\title{
Simulation of semi-explicit mechanisms of SOA formation from glyoxal in aerosol in a 3-D model
}

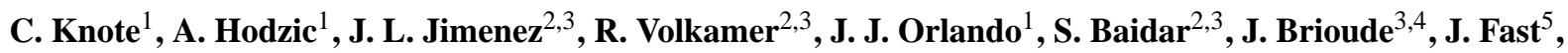 \\ D. R. Gentner ${ }^{6}$, A. H. Goldstein ${ }^{6,7}$, P. L. Hayes ${ }^{2,3}$, W. B. Knighton ${ }^{8}$, H. Oetjen ${ }^{2}$, A. Setyan ${ }^{9}$, H. Stark ${ }^{10,3}$, \\ R. Thalman ${ }^{2,3}$, G. Tyndall ${ }^{1}$, R. Washenfelder ${ }^{3,4}$, E. Waxman ${ }^{2,3}$, and Q. Zhang ${ }^{9}$ \\ ${ }^{1}$ Atmospheric Chemistry Division, National Center for Atmospheric Research, Boulder, CO, USA \\ ${ }^{2}$ Department of Chemistry and Biochemistry, University of Colorado, Boulder, CO, USA \\ ${ }^{3}$ CIRES, University of Colorado, Boulder, CO, USA \\ ${ }^{4}$ Chemical Sciences Division, NOAA Earth System Research Laboratory, Boulder, CO, USA \\ ${ }^{5}$ Pacific Northwest National Laboratory, Richland, Washington, CO, USA \\ ${ }^{6}$ Dept. of Civil \& Environmental Engineering, University of California, Berkeley, CA, USA \\ ${ }^{7}$ Dept. of Environmental Science, Policy, and Management, University of California, Berkeley, CA, USA \\ ${ }^{8}$ Montana State University, Bozeman, MT, USA \\ ${ }^{9}$ Department of Environmental Toxicology, University of California, Davis, USA \\ ${ }^{10}$ Aerodyne Research, Inc., Billerica, MA, USA
}

Correspondence to: A. Hodzic (alma@ucar.edu)

Received: 19 July 2013 - Published in Atmos. Chem. Phys. Discuss.: 15 October 2013

Revised: 1 May 2014 - Accepted: 20 May 2014 - Published: 24 June 2014

\begin{abstract}
New pathways to form secondary organic aerosol (SOA) have been postulated recently. Glyoxal, the smallest dicarbonyl, is one of the proposed precursors. It has both anthropogenic and biogenic sources, and readily partitions into the aqueous phase of cloud droplets and deliquesced particles where it undergoes both reversible and irreversible chemistry. In this work we extend the regional scale chemistry transport model WRF-Chem to include detailed gasphase chemistry of glyoxal formation as well as a state-ofthe-science module describing its partitioning and reactions in the aerosol aqueous-phase. A comparison of several proposed mechanisms is performed to quantify the relative importance of different formation pathways and their regional variability. The CARES/CalNex campaigns over California in summer 2010 are used as case studies to evaluate the model against observations. A month-long simulation over the continental United States (US) enables us to extend our results to the continental scale.

In all simulations over California, the Los Angeles (LA) basin was found to be the hot spot for SOA formation from glyoxal, which contributes between $1 \%$ and $15 \%$ of the model SOA depending on the mechanism used. Our results
\end{abstract}

indicate that a mechanism based only on a reactive (surface limited) uptake coefficient leads to higher SOA yields from glyoxal compared to a more detailed description that considers aerosol phase state and chemical composition. In the more detailed simulations, surface uptake is found to give the highest SOA mass yields compared to a volume process and reversible formation. We find that the yields of the latter are limited by the availability of glyoxal in aerosol water, which is in turn controlled by an increase in the Henry's law constant depending on salt concentrations ("salting-in"). A time dependence in this increase prevents substantial partitioning of glyoxal into aerosol water at high salt concentrations. If this limitation is removed, volume pathways contribute $>20 \%$ of glyoxal-SOA mass, and the total mass formed ( $5.8 \%$ of total SOA in the LA basin) is about a third of the simple uptake coefficient formulation without consideration of aerosol phase state and composition. Results from the continental US simulation reveal the much larger potential to form glyoxal-SOA over the eastern continental US. Interestingly, the low concentrations of glyoxal-SOA over the western continental US are not due to the lack of a potential to form glyoxal-SOA here. Rather these small glyoxal-SOA 
concentrations reflect dry conditions and high salt concentrations, and the potential to form SOA mass here will strongly depend on the water associated with particles.

\section{Introduction}

Organic matter is a major contributor to atmospheric aerosol load. While a fraction of it is directly emitted as primary particles into the air, a substantial contribution - often the majority - is generated within the atmosphere through chemistry. The progressive oxidation of precursor gases (volatile organic compounds, VOCs) leads to semivolatile products that have lower volatility and higher water solubility than their parent precursors, and can form secondary organic aerosol (SOA). The large number of organic compounds involved proves to be challenging for both measurement and modeling communities (e.g., Goldstein and Galbally, 2007). Recent studies further indicate that SOA formation in the aqueous phase may be important (Lim et al., 2010; Ervens et al., 2011). Here we investigate SOA formation from glyoxal in or on deliquesced particles. Glyoxal is the smallest dicarbonyl produced by oxidation of both anthropogenic and biogenic precursors. It is short-lived, so it can serve as an indicator for recent VOC oxidation processes in the atmosphere (Volkamer et al., 2005). Laboratory measurements for glyoxal confirmed that it partitions into the particle phase under atmospheric conditions due to its high solubility in water and its subsequent aqueous-phase chemistry (Kroll et al., 2005; Liggio et al., 2005; Carlton et al., 2007; Noziere et al., 2009; Galloway et al., 2009; Volkamer et al., 2009). In particular, recent work has shown that concentrated salt solutions strongly shift the partitioning of glyoxal towards the liquidphase (Kampf et al., 2013), where it is a source for SOA with a high oxygen-to-carbon ratio (Waxman et al., 2013). Finally, Holzinger et al. (2013) found that a multi-phase process might be needed to explain the aerosol nitrogen content in the very region and time period we will focus on.

Formation of SOA from glyoxal has been studied with box models (Volkamer et al., 2007; Lim et al., 2010; McNeill et al., 2012; Waxman et al., 2013) and with a 1-D transport model employing the Master Chemical Mechanism (Washenfelder et al., 2011) with the difference between measured and modeled glyoxal concentrations attributed to an aerosol loss process. Volkamer et al. (2007) find that glyoxal could contribute up to $15 \%$ to total SOA in Mexico City for the period investigated. Washenfelder et al. (2011) however derive lower uptake coefficients over California and conclude that glyoxal contribution to total SOA is in the range of $0-4 \%$. Uptake coefficients derived by Liggio et al. (2005) were used in global 3-D simulations with simplified chemistry (Fu et al., 2008) where they found that glyoxal contributes additional $2.6 \mathrm{Tg} \mathrm{Ca}^{-1}$ to the global SOA source of $17.5 \mathrm{Tg} \mathrm{Ca}^{-1}$ from a traditional mechanism $(\sim 13 \%)$. The partitioning behavior was hence already studied at urban and global scales. Previous box-model studies were locally constrained by observations but were run in idealized conditions and lack most of the variability due to emissions, transport and chemistry found at the regional scale. Global-scale 3-D simulations on the other hand lack the degree of detail in their description of the glyoxal atmospheric processing necessary for accurate simulations. Most previous studies did not consider aerosol properties other than surface area, or included reversible partitioning of glyoxal into deliquesced droplets. $\mathrm{Li}$ et al. (2013) investigated formation of SOA from deliquesced particles and cloud droplets on the regional scale in the Pearl River Delta with a simple surface uptake parameterization. Waxman et al. (2013) is the only study that incorporated recent findings regarding the "salting-in" of glyoxal, that is, the strong ( $\sim 3$ orders of magnitude) increase in the effective Henry's law constant due to glyoxal interactions with salts (Kampf et al., 2013). They concluded that the catalytic reaction of $\mathrm{NH}_{4}^{+}$(Noziere et al., 2009) exceeds reactions by $\mathrm{OH}$ radicals to form SOA from glyoxal, and pointed out the importance of particle $\mathrm{pH}$, and phase state for the rate of SOA formation from glyoxal. There is no previous study of glyoxal-SOA formation within deliquesced particles incorporating these findings in a 3-D regional model. A model capable of representing detailed aerosol characteristics, like $\mathrm{pH}$, size resolved chemical composition, and phase state could provide valuable information to assess which parameters determine the regional variability of glyoxal SOA.

In this study we investigate the life cycle of glyoxal in the atmosphere at the regional scale over California and the continental scale over the United States. We choose summer 2010 as our study period as the Carbonaceous Aerosols and Radiative Effects Study (CARES, Zaveri et al., 2012) and the Research at the Nexus of Air Quality and Climate Change (CalNex, Ryerson et al., 2013) campaigns took place in California during that time, and provide measurements that allow constraining glyoxal sources, concentrations, and sinks. A number of studies using this data set investigated SOA formation (Duong et al., 2011; Ahlm et al., 2012; Gentner et al., 2012; Liu et al., 2012a, b; O'Brien et al., 2012; Rollins et al., 2012; Zhang et al., 2012; Hayes et al., 2013; Hersey et al., 2013; Setyan et al., 2012; Shilling et al., 2013; Zhao et al., 2013), the oxidative environment (Pusede and Cohen, 2012; Warneke et al., 2013) and emissions of compounds related to SOA formation like VOCs (Borbon et al., 2013), $\mathrm{NO}_{\mathrm{x}}$ (McDonald et al., 2012; Brioude et al., 2013; Baidar et al., 2013b), or ammonia (Nowak et al., 2012). We employ the mesoscale chemistry transport model WRF-chem (Grell et al., 2005) which we extended to include a more detailed description of gas-phase chemistry producing glyoxal as well as a new module to describe its partitioning into the aerosol phase. In our study, substantial effort has been put into improving existing emission inventories for the California domain, and in evaluating the model against measurements. Several pathways to form SOA from glyoxal within 
Table 1. Chosen parameterization for selected physical processes in WRF.

\begin{tabular}{ll}
\hline Process & Method \\
\hline Cloud microphysics & Morrison double-moment scheme \\
Radiation & RRTMG short- and longwave \\
Land surface & Noah Land Surface Model \\
Urban surface & Urban Canopy Model \\
Planetary boundary layer & Mellor-Yamada Nakanishi and Niino 2.5 \\
Cumulus parameterization & Grell 3-D ensemble \\
\hline
\end{tabular}

deliquesced particles were added, including reversible partitioning. We present results from a number of simulations over California in which we considered (combinations of) several approaches to this partitioning, and investigate relative contributions and their regional variability. We further show the importance of these processes on the continental scale. In Sect. 2 we describe the modeling system, including updates made to emissions and gas-phase chemistry, and the modeling of glyoxal partitioning into the particle phase. Section 3 shows how our simulations compare against measurements made during the CARES/CalNex campaigns, and emphasizes the improvements due to the updated emissions inventory. In Sect. 4 we investigate the partitioning behavior of glyoxal based on the different pathways and its regional variability, and present the result of the continental-scale simulation.

\section{Modeling}

\subsection{Model setup}

Simulations and developments are based on the Weather Research and Forecasting Model coupled with Chemistry (WRF-chem) (Grell et al., 2005; Fast et al., 2006) in version 3.4.1. Table 1 lists the options chosen for physical parameterizations. The chemical mechanism of the Model for Ozone and Related Chemical Tracers, version 4 (MOZART4, Emmons et al., 2010) is employed to calculate gas-phase chemical reactions, with the fast Tropospheric UltravioletVisible (fTUV) module (Tie et al., 2003) used for calculating photolysis rates. Aerosol dynamics and associated physical and chemical processes are represented by the Model for Simulating Aerosol Interactions and Chemistry (MOSAIC, Zaveri et al., 2008) using four size bins (39-156 nm, 156$625 \mathrm{~nm}, 625-2.5 \mu \mathrm{m}, 2.5-10 \mu \mathrm{m})$. Inorganic aerosol thermodynamics within MOSAIC are solved using the multicomponent equilibrium solver for aerosols (MESA, Zaveri et al., 2005). Secondary organic aerosol (SOA) formation from anthropogenic VOCs and semi/intermediate volatility compounds ( $\mathrm{S} / \mathrm{IVOCs}$ ) is parameterized with the method of Hodzic and Jimenez (2011), where an SOA is formed based on a tracer co-emitted with carbon monoxide. By reaction with $\mathrm{OH}$ this tracer is then converted into an SOA. Primary

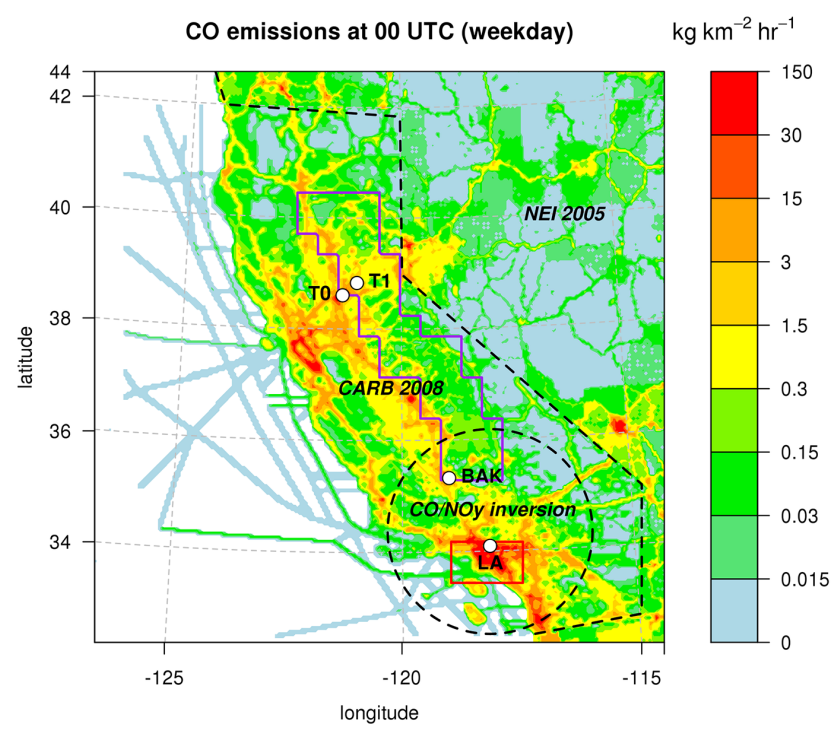

Figure 1. Model domain and schematic illustrating the emissions merging (see text), with $\mathrm{CO}$ emissions $\left(\mathrm{kg} \mathrm{km}^{-2} \mathrm{hr}^{-1}\right)$ as visual guideline in the background. Measurement "supersites" from CARES and CalNex shown: T0 American River College, Sacramento. T1 Northside School, Cool. BAK Cooperative Extension Kern County, Bakersfield. LA California Institute of Technology campus, Pasadena. Two focus regions are marked: the LA basin (red, in the following called "LA"), and the isoprene-rich eastern slopes of the Central Valley (purple, called "ESCV").

organic aerosol is considered to be inert, evaporation and subsequent oxidation and recondensation is not considered. To account for this we lowered the SOA formation yields from $80 \mu \mathrm{g} \mathrm{m}^{-3} \mathrm{ppmv}^{-1}$ to $40 \mu \mathrm{g} \mathrm{m}^{-3} \mathrm{ppmv}^{-1}$. Treatment of biogenic SOA follows Shrivastava et al. (2011).

The regional model domain (Fig. 1) covers the state of California including adjacent states and parts of the Pacific Ocean, and is represented as a $280 \times 280 \times 40$ cell grid on a Lambert conformal conic projection with a horizontal resolution of $4 \mathrm{~km}$, extending vertically up to $10 \mathrm{hPa}$. Our simulations over this region are conducted for the period of 29 May until 15 June 2010. The simulation at the continental scale covers the continental United States (CONUS) at a horizontal resolution of $36 \mathrm{~km}$ and spans the whole month of July 2010.

All simulations are initialized and forced at the lateral boundaries by 6 hourly analysis data from the NCEP Global Forecast System (GFS) at $1.0^{\circ}$ resolution for meteorology (NCEP, 2000), and 6 hourly MOZART-GEOS5 simulations (based on Emmons et al., 2010) at $1.9 \times 2.5^{\circ}$ resolution for chemistry and aerosol. Simulations are nudged to GFS analyses above the boundary layer throughout the simulation. Sea surface temperature (SST) analyses updated every 6 hours from the US Navy Fleet Numerical Meteorology and Oceanography Center (http://www.usgodae.org/ftp/ outgoing/fnmoc/models/ghrsst/docs/ghrsst_doc.txt) are used 
Table 2. Emissions scaling factors $\left(E=E_{\text {orig }} \cdot\right.$ Scaling Factor $)$, for 2010 relative to $2005 / 2008$.

\begin{tabular}{lc}
\hline Species & Scaling factor \\
\hline $\mathrm{SO}_{2}$ & 0.55 \\
$\mathrm{NO}, \mathrm{NO}_{2}$ & 0.8 \\
$\mathrm{CO}$ & 1.2 \\
$\mathrm{NH}_{3}$ & 1.0 \\
glyoxal, methylglyoxal & 1.0 \\
all other gaseous emissions & 0.65 \\
all aerosol emissions & 1.0
\end{tabular}

instead of default climatologies. The simulations were conducted using the National Center for Atmospheric Research (NCAR) high performance computing system "Yellowstone" (CISL , 2012).

\subsection{Emissions}

Anthropogenic emission estimates over California were created as a combination of the National Emission Inventory data set for 2005 (NEI 2005), the California Air Resources Board emission estimates for 2008 (CARB 2008), the inversion-based $\mathrm{CO}$ and $\mathrm{NO}_{\mathrm{x}}$ emissions for the LA basin as presented by Brioude et al. (2013), and VOC/CO emission ratios of Borbon et al. (2013). The following steps are applied to create the final anthropogenic emission data set (see also Fig. 1). The NEI 2005 emissions data set was used as basis. Over California, it is first replaced by the CARB 2008 inventory. VOCs in both the NEI and CARB inventory were originally speciated for the SAPRC99 mechanism (Carter et al., 2000), and were translated to species available in MOZART. Emissions are then reduced by $35 \%$ for most species to account for the changes between 2005/2008 and 2010 (Table 2). $\mathrm{CO}$ and $\mathrm{NO}_{\mathrm{x}}$ emission amounts for grid points in the LA basin are replaced with inversion estimates from Brioude et al. (2013).

VOC/CO emission ratios reported by Borbon et al. (2013) were used to create VOC emission fields for selected VOCs, using the spatial distribution and temporal behavior of the $\mathrm{CO}$ emissions created as described above. These relationships were derived from measurements in the Los Angeles basin during 2010, and are more recent and considered more accurate than estimates from the CARB and NEI inventories. We replaced the VOC emission estimates from the inventories with our inversion based inventory for the species available in Borbon et al. (2013). Figure 2 shows that there are considerable differences in the estimates of speciated VOC emissions between the CARB inventory and the amounts estimated from the $\mathrm{CO}$ inversion and the measured $\mathrm{VOC} / \mathrm{CO}$ emission ratios in the LA basin. An investigation of these differences would be beneficial for the modeling community, but is out of the scope of this work.

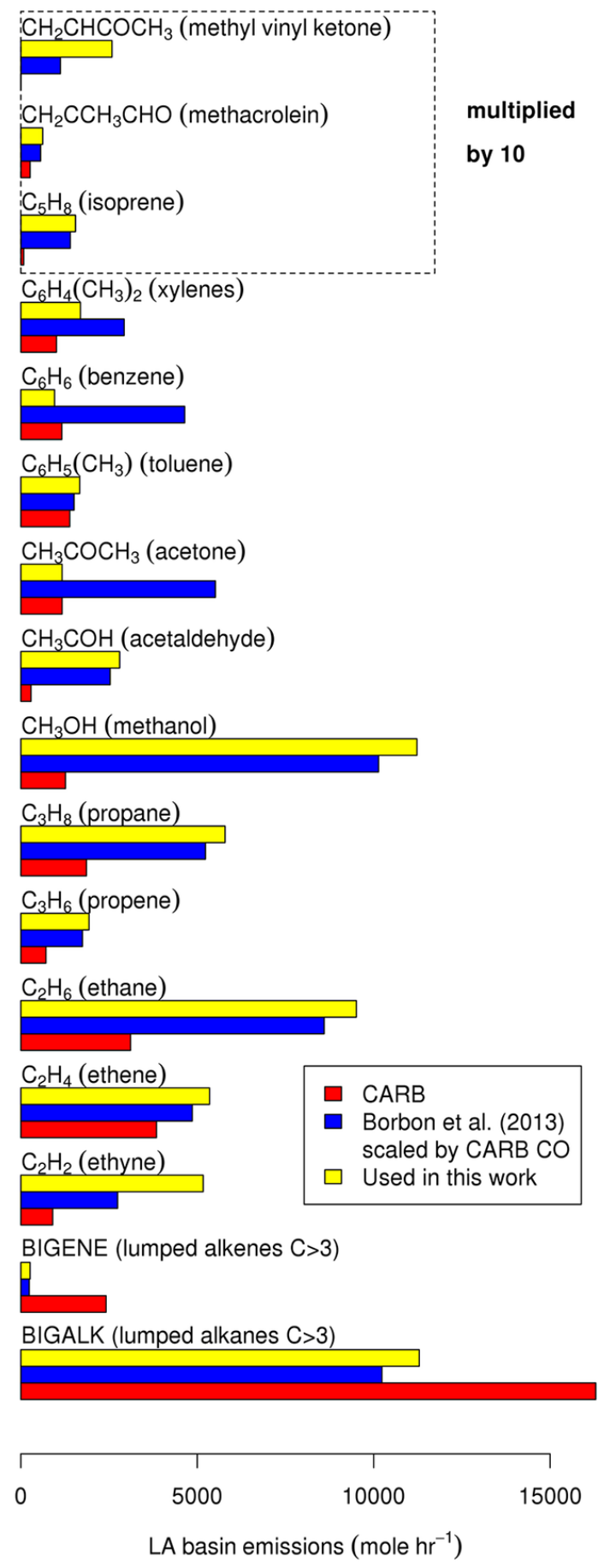

Figure 2. VOC emissions in the LA basin (rectangular box from $33.6^{\circ} \mathrm{N}, 119.0^{\circ} \mathrm{W}$, to $34.3^{\circ} \mathrm{N}, 117.7^{\circ} \mathrm{W}$ ) for selected MOZART-4 species as given by the CARB 2008 inventory (red), scaling factors in Borbon et al. (2013) multiplied by CARB 2008 CO emissions (blue), and the final emissions used, a result of the emissions preparation procedure outlined in the text (yellow). 
The final emissions used in our simulations over California are a result of an iterative approach to reconcile simulated concentrations with observations. Note that for acetone, benzene, and xylenes, the emission amounts based on COVOC ratios from Borbon et al. (2013) are much higher than reported in CARB. Simulations made with these inversionbased amounts showed strongly overestimated concentrations compared to surface measurements. Hence we reduced these emissions so that simulated concentrations agreed with measurements. Interestingly, the resulting emission amounts for these species were very similar to the ones reported in the inventory. A comparison of a simulation using the original emissions as given from the combination of NEI/CARB inventories and our updated inventory against measurement data is discussed in Sect. 3.

Biogenic emissions from vegetation are calculated as a function of ambient conditions with the Model of Emissions of Gases and Aerosols from Nature (MEGAN) version 2.04 (Guenther et al., 2006). During evaluation we found a large underestimation of isoprene compared to measurements in urban areas like the LA basin (LA ground site), while no such underestimation was found in rural environments (Bakersfield, T1 sites). We attribute this to an insufficient description of vegetation in urban areas in the MEGAN land use database (A. Guenther, NCAR, personal communication, 2012) and have hence increased emissions of all biogenic VOCs as determined in MEGAN by a factor of 2.5 over grid points with an "urban" land use type in WRF-chem.

For the CONUS simulation we used anthropogenic emissions from the National Emission Inventory for 2008 (NEI 2008) updated by the U.S. Environmental Protection Agency (EPA) to reflect emissions of the year 2010 within the Air Quality Model Evaluation International Initiative (AQMEII, http://aqmeii.jrc.ec.europa.eu) without further modifications. Biogenic emissions from MEGAN were used without the modifications for the simulations over California described above.

\subsection{The modeled glyoxal life cycle}

The MOZART mechanism describes the formation of glyoxal in the gas phase. Direct anthropogenic emissions of glyoxal are included according to the CARB emissions inventory, but were found to be negligible $(<0.1 \%$ contribution to average surface glyoxal production), which is consistent with earlier findings based on ambient time-series analysis in Mexico City (Volkamer et al., 2005). Glyoxal is removed from the atmosphere by photolysis, oxidation through reaction with the $\mathrm{OH}$ radical, dry deposition and through partitioning into particles. A detailed description of the aerosol sink for glyoxal follows in section 2.3.2, and the reader is invited to consult Figure 14 showing the effect of this sink on gas-phase glyoxal concentrations. A constant dry deposition velocity of $2 \mathrm{~mm} \mathrm{~s}^{-1}$ is used as derived in Washenfelder et al. (2011) based on nighttime decreases of glyoxal at the LA ground site. Wet deposition is not considered.

\subsubsection{Updates to the MOZART-4 gas-phase mechanism}

Table 3 lists new and updated species, Table 4 the new and altered reactions, and Table 5 the added photolysis reactions. All changes presented are relative to the mechanism described in Emmons et al. (2010).

Formation of glyoxal in the gas-phase is described in the MOZART mechanism, which already treats glyoxal as an explicit species (Emmons et al., 2010). Four main formation pathways are considered: oxidation of isoprene, of aromatic compounds, of MBO (2-methyl-3-buten-2-ol), and of ethyne. We updated the description of isoprene oxidation in MOZART by adding a $2 \%$ molar yield of each glyoxal, methylglyoxal, glycolaldehyde, and hydroxyacetone from the reaction of the isoprene peroxy radical with $\mathrm{NO}$ and with $\mathrm{NO}_{3}$, the former having recently been observed in laboratory experiments (Volkamer et al., 2005; Galloway et al., 2011, Thalman et al., 2013). We further included a more explicit treatment of C5 unsaturated hydroxycarbonyl chemistry, a potential additional secondary glyoxal source. Note, however, that while this formation of glyoxal is expected theoretically, it has not been observed in recent laboratory experiments (Thalman et al., 2013). We have further speciated the aromatics (previously lumped as one surrogate) into benzene, toluene and lumped isomers of xylenes with corresponding glyoxal yields for each precursor (Volkamer et al., 2001; Calvert et al., 2002). The treatment of these aromatic species is taken from the Leeds Master Chemical Mechanism (Bloss et al., 2005, http://mcm.leeds.ac.uk/MCM/), although the chemistry of some later-generation products is ignored as these multi-functional species are likely prone to heterogeneous removal, are oxidized far from the source region, and/or are not significant sources of glyoxal. MBO chemistry, initiated via reaction with $\mathrm{OH}, \mathrm{NO}_{3}$ and ozone, is also added to the model. Note that glycolaldehyde is a significant product of the $\mathrm{OH}$-initiated oxidation of $\mathrm{MBO}$, and that further reactions of glycolaldehyde lead to glyoxal production. Ethyne has also been added as an explicit species and its oxidation chemistry is included; the rate coefficient for its reaction with $\mathrm{OH}$ is from the JPL evaluation (Sander et al., 2011) and products are from Hatakeyama et al. (1986). $\mathrm{HONO}$ is now considered as an additional source of $\mathrm{OH}$ radicals through photolysis.

\subsubsection{Pathways of interaction between gas-phase glyoxal and particles}

There is no clear scientific consensus on how glyoxal might form SOA. Irreversible SOA formation via both a surface uptake and volume reactions have been proposed (Ervens and Volkamer, 2010, and references therein). Reversible partitioning and oligomerization reactions are observed in 
Table 3. New/updated chemical species in MOZART-4.

\begin{tabular}{|c|c|c|}
\hline Symbolic name & Atomic composition & Comments \\
\hline $\mathrm{C} 2 \mathrm{H} 2$ & $\mathrm{C}_{2} \mathrm{H}_{2}$ & ethyne \\
\hline $\mathrm{HCOOH}$ & $\mathrm{HCOOH}$ & formic acid \\
\hline $\mathrm{HOCH} 2 \mathrm{OO}$ & $\mathrm{HOCH}_{2} \mathrm{OO}$ & $\begin{array}{l}\text { radical formed in } \mathrm{HO}_{2} \text { addition } \\
\text { to formaldehyde }\end{array}$ \\
\hline HONO & HONO & \\
\hline TOLUENE & $\mathrm{C}_{6} \mathrm{H}_{5}\left(\mathrm{CH}_{3}\right)$ & $\begin{array}{l}\text { unlumped, represented sum of benzene } \\
+ \text { toluene }+ \text { xylenes in old version }\end{array}$ \\
\hline BENZENE & $\mathrm{C}_{6} \mathrm{H}_{6}$ & \\
\hline PHENOL & $\mathrm{C}_{6} \mathrm{H}_{5} \mathrm{OH}$ & \\
\hline BEPOMUC & $\mathrm{C}_{6} \mathrm{H}_{6} \mathrm{O}_{3}$ & Unsaturated epoxide-dialdehyde \\
\hline BENZO2 & $\mathrm{C}_{6} \mathrm{H}_{6}(\mathrm{OH})(\mathrm{OO})_{2}$ & $\begin{array}{l}\text { Bicyclic peroxy radical from } \\
\text { OH addition to benzene }\end{array}$ \\
\hline PHENO2 & $\mathrm{C}_{6} \mathrm{H}_{5}(\mathrm{OH})_{2}(\mathrm{OO})_{2}$ & $\begin{array}{l}\text { Bicyclic peroxy radical from } \\
\text { OH addition to phenol }\end{array}$ \\
\hline PHENO & $\mathrm{C}_{6} \mathrm{H}_{5}(\mathrm{OH})_{2}(\mathrm{OO}) \mathrm{O}$ & $\begin{array}{l}\text { Bicyclic oxy radical from } \mathrm{OH} \\
\text { addition to phenol }\end{array}$ \\
\hline PHENOOH & $\mathrm{C}_{6} \mathrm{H}_{5}(\mathrm{OH})_{2}(\mathrm{OO}) \mathrm{OOH}$ & $\begin{array}{l}\text { Bicyclic hydroperoxide from } \mathrm{OH} \\
\text { addition to phenol }\end{array}$ \\
\hline $\mathrm{C} 6 \mathrm{H} 5 \mathrm{O} 2$ & $\mathrm{C}_{6} \mathrm{H}_{5} \mathrm{O}_{2}$ & \\
\hline $\mathrm{C} 6 \mathrm{H} 5 \mathrm{OOH}$ & $\mathrm{C}_{6} \mathrm{H}_{5} \mathrm{OOH}$ & \\
\hline BENZOOH & $\mathrm{C}_{6} \mathrm{H}_{6}(\mathrm{OH})(\mathrm{OO}) \mathrm{OOH}$ & $\begin{array}{l}\text { Bicyclic hydroperoxide from } \mathrm{OH} \\
\text { addition to benzene }\end{array}$ \\
\hline BIGALD1 & $\mathrm{HC}(=\mathrm{O}) \mathrm{CH}=\mathrm{CHCH}=\mathrm{O}$ & Unsaturated dialdehyde \\
\hline BIGALD2 & $\mathrm{CH}_{3} \mathrm{C}(=\mathrm{O}) \mathrm{CH}=\mathrm{CHCH}=\mathrm{O}$ & Unsaturated dicarbonyl \\
\hline BIGALD3 & $\mathrm{HC}(=\mathrm{O}) \mathrm{C}\left(\mathrm{CH}_{3}\right)=\mathrm{CHCH}=\mathrm{O}$ & Unsaturated dialdehyde \\
\hline BIGALD4 & $\begin{array}{l}\mathrm{CH}_{3} \mathrm{C}(=\mathrm{O}) \mathrm{CH}=\mathrm{C}\left(\mathrm{CH}_{3}\right) \mathrm{CH}=\mathrm{O} \\
\text { (and isomers) }\end{array}$ & $\begin{array}{l}\text { Unsaturated dicarbonyls from } \\
\text { xylene oxidation }\end{array}$ \\
\hline MALO2 & $\mathrm{HC}(=\mathrm{O}) \mathrm{CH}=\mathrm{CHC}(=\mathrm{O}) \mathrm{OO}$ & $\begin{array}{l}\text { Acyl radical from "BIGALD1" } \\
\text { photolysis }\end{array}$ \\
\hline PBZNIT & $\mathrm{C}_{6} \mathrm{H}_{5} \mathrm{C}(\mathrm{O}) \mathrm{OONO}_{2}$ & Peroxybenzoyl nitrate \\
\hline TEPOMUC & $\mathrm{C}_{7} \mathrm{H}_{8} \mathrm{O}_{3}$ & Unsaturated epoxide-dialdehyde \\
\hline $\mathrm{BZOO}$ & $\mathrm{C}_{6} \mathrm{H}_{5} \mathrm{CH}_{2} \mathrm{O}_{2}$ & $\begin{array}{l}\text { Peroxy radical formed following } \mathrm{OH} \\
\text { abstraction from toluene }\end{array}$ \\
\hline BZOOH & $\mathrm{C}_{6} \mathrm{H}_{5} \mathrm{CH}_{2} \mathrm{OOH}$ & \\
\hline BZALD & $\mathrm{C}_{6} \mathrm{H}_{5} \mathrm{CHO}$ & Benzaldehyde \\
\hline $\mathrm{ACBZO} 2$ & $\mathrm{C}_{6} \mathrm{H}_{5} \mathrm{C}(\mathrm{O}) \mathrm{OO}$ & $\begin{array}{l}\text { Acylperoxy radical obtained from } \\
\text { benzaldehyde }\end{array}$ \\
\hline DICARBO2 & $\begin{array}{l}\mathrm{CH}_{3} \mathrm{C}(=\mathrm{O}) \mathrm{CH}=\mathrm{C}\left(\mathrm{CH}_{3}\right) \mathrm{C}(=\mathrm{O}) \mathrm{OO} \\
\text { and isomers }\end{array}$ & $\begin{array}{l}\text { Acylperoxy radical obtained from } \\
\text { photolysis of unsaturated dicarbonyls }\end{array}$ \\
\hline MDIALO2 & $\begin{array}{l}\mathrm{HC}(=\mathrm{O}) \mathrm{C}\left(\mathrm{CH}_{3}\right)=\mathrm{CHC}(=\mathrm{O}) \mathrm{OO} \\
\text { and isomer }\end{array}$ & $\begin{array}{l}\text { Acylperoxy radical obtained from } \\
\text { photolysis of unsaturated dicarbonyls }\end{array}$ \\
\hline XYLENES & $\mathrm{C}_{6} \mathrm{H}_{4}\left(\mathrm{CH}_{3}\right)_{2}$ & Lumped isomers of xylene \\
\hline XYLOL & Isomers of $\mathrm{C}_{6} \mathrm{H}_{3}\left(\mathrm{CH}_{3}\right)_{2}(\mathrm{OH})$ & \\
\hline XYLOLO2 & $\begin{array}{l}\text { Isomers of } \\
\mathrm{C}_{6} \mathrm{H}_{3}\left(\mathrm{CH}_{3}\right)_{2}(\mathrm{OH})_{2}(\mathrm{OO})_{2}\end{array}$ & $\begin{array}{l}\text { Bicyclic peroxy radical from } \mathrm{OH} \\
\text { addition to xylenols }\end{array}$ \\
\hline XYLOLOOH & $\begin{array}{l}\text { Isomers of } \\
\mathrm{C}_{6} \mathrm{H}_{3}\left(\mathrm{CH}_{3}\right)_{2}(\mathrm{OH})_{2}(\mathrm{OO}) \mathrm{OOH}\end{array}$ & $\begin{array}{l}\text { Bicyclic hydroperoxide from } \mathrm{OH} \\
\text { addition to xylenols }\end{array}$ \\
\hline XYLENO2 & $\begin{array}{l}\text { Isomers of } \\
\mathrm{C}_{6} \mathrm{H}_{4}\left(\mathrm{CH}_{3}\right)_{2}(\mathrm{OH})(\mathrm{OO})_{2}\end{array}$ & $\begin{array}{l}\text { Bicyclic peroxy radicals from } \mathrm{OH} \\
\text { addition to xylenes }\end{array}$ \\
\hline XYLENOOH & $\begin{array}{l}\text { Isomers of } \\
\mathrm{C}_{6} \mathrm{H}_{4}\left(\mathrm{CH}_{3}\right)_{2}(\mathrm{OH})(\mathrm{OO}) \mathrm{OOH}\end{array}$ & $\begin{array}{l}\text { Bicyclic hydroperoxides from } \mathrm{OH} \\
\text { addition to xylenes }\end{array}$ \\
\hline MBO & $\mathrm{CH}_{2}=\mathrm{CH}-\mathrm{C}(\mathrm{OH})\left(\mathrm{CH}_{3}\right)_{2}$ & 2-methyl-3-buten-2-ol \\
\hline MBOO2 & $\mathrm{HOCH}_{2}-\mathrm{CH}(\mathrm{OO})-\mathrm{C}(\mathrm{OH})\left(\mathrm{CH}_{3}\right)_{2}$ & Peroxy radical from $\mathrm{OH}+\mathrm{MBO}$ \\
\hline MBONO3O2 & $\mathrm{O}_{2} \mathrm{NOCH}_{2}-\mathrm{CH}(\mathrm{OO})-\mathrm{C}(\mathrm{OH})\left(\mathrm{CH}_{3}\right)_{2}$ & Peroxy radical from $\mathrm{NO}_{3}+\mathrm{MBO}$ \\
\hline $\mathrm{MBOOOH}$ & $\mathrm{HOCH}_{2}-\mathrm{CH}(\mathrm{OOH})-\mathrm{C}(\mathrm{OH})\left(\mathrm{CH}_{3}\right)_{2}$ & $\begin{array}{l}\text { Dihydroxy-hydroperoxide formed } \\
\text { in reaction of } \mathrm{MBOO} 2 \text { with } \mathrm{HO}_{2}\end{array}$ \\
\hline HMPROP & $\left(\mathrm{CH}_{3}\right)_{2} \mathrm{C}(\mathrm{OH}) \mathrm{CH}=\mathrm{O}$ & 2-hydroxy-2-methyl-propanal \\
\hline HMPROPO2 & $\left(\mathrm{CH}_{3}\right)_{2} \mathrm{C}(\mathrm{OH}) \mathrm{C}(\mathrm{O}) \mathrm{OO}$ & $\begin{array}{l}\text { Peroxy radical formed from } \\
\mathrm{OH}+\mathrm{HMPROP} \text { reaction }\end{array}$ \\
\hline
\end{tabular}


Table 4. New/updated gas-phase reactions. ( $\{\mathrm{CO} 2\}$ indicates $\mathrm{CO}_{2}$ is not included in the model solution.)

\begin{tabular}{|c|c|c|c|}
\hline Reactants & & Products & Rate constant(s) \\
\hline $\mathrm{C} 2 \mathrm{H} 2+\mathrm{OH}$ & $\rightarrow$ & $.65 \cdot \mathrm{GLYOXAL}+.65 \cdot \mathrm{OH}+.35 \cdot \mathrm{HCOOH}+.35 \cdot \mathrm{HO} 2+.35 \cdot \mathrm{CO}$ & $\begin{array}{l}\text { ko }=5.5 \mathrm{E}-30 \\
\mathrm{ki}=8.3 \times 10^{-13} \cdot(300 / \mathrm{T})^{-2} ; \mathrm{f}=0.6\end{array}$ \\
\hline $\mathrm{HCOOH}+\mathrm{OH}$ & $\rightarrow$ & $\mathrm{HO} 2+\mathrm{H} 2 \mathrm{O}+\{\mathrm{CO} 2\}$ & $4.5 \times 10^{-13}$ \\
\hline $\mathrm{CH} 2 \mathrm{O}+\mathrm{HO} 2$ & $\rightarrow$ & $\mathrm{HOCH} 2 \mathrm{OO}$ & $9.7 \times 10^{-15} \cdot \exp (625 / \mathrm{T})$ \\
\hline HOCH2OO & $\rightarrow$ & $\mathrm{CH} 2 \mathrm{O}+\mathrm{HO} 2$ & $2.4 \mathrm{E} 12 \cdot \exp (-7000 / \mathrm{T})$ \\
\hline $\mathrm{HOCH} 2 \mathrm{OO}+\mathrm{NO}$ & $\rightarrow$ & $\mathrm{HCOOH}+\mathrm{NO} 2+\mathrm{HO} 2$ & $2.6 \times 10^{-12} \cdot \exp (265 / \mathrm{T})$ \\
\hline $\mathrm{HOCH} 2 \mathrm{OO}+\mathrm{HO} 2$ & $\rightarrow$ & $\mathrm{HCOOH}$ & $7.5 \times 10^{-13} \cdot \exp (700 / \mathrm{T})$ \\
\hline ISOPO $2+\mathrm{NO}$ & $\rightarrow$ & $\begin{array}{l}.08 \cdot \mathrm{ONITR}+.92 \cdot \mathrm{NO} 2+.23 \cdot \mathrm{MACR}+.32 \cdot \mathrm{MVK} \\
+.33 \cdot \mathrm{HYDRALD}+.02 \cdot \mathrm{GLYOXAL}+.02 \cdot \mathrm{GLYALD} \\
+.02 \cdot \mathrm{CH} 3 \mathrm{COCHO}+.02 \cdot \mathrm{HYAC}+.55 \cdot \mathrm{CH} 2 \mathrm{O}+.92 \cdot \mathrm{HO} 2\end{array}$ & $4.4 \times 10^{-12} \cdot \exp (180 / \mathrm{T})$ \\
\hline ISOPO2+NO3 & $\rightarrow$ & $\begin{array}{l}\mathrm{HO} 2+\mathrm{NO} 2+.6 \cdot \mathrm{CH} 2 \mathrm{O}+.25 \cdot \mathrm{MACR}+.35 \cdot \mathrm{MVK} \\
+.36 \cdot \mathrm{HYDRALD}+.02 \cdot \mathrm{HYAC}+.02 \cdot \mathrm{CH} 3 \mathrm{COCHO} \\
+.02 \cdot \mathrm{GLYOXAL}+.02 \cdot \mathrm{GLYALD}\end{array}$ & $2.4 \times 10^{-12}$ \\
\hline $\mathrm{XO} 2+\mathrm{NO}$ & $\rightarrow$ & $\begin{array}{l}\mathrm{NO} 2+\mathrm{HO} 2+.25 \cdot \mathrm{CO}+.25 \cdot \mathrm{CH} 2 \mathrm{O}+.25 \cdot \mathrm{GLYOXAL} \\
+.25 \cdot \mathrm{CH} 3 \mathrm{COCHO}+.25 \cdot \mathrm{HYAC}+.25 \cdot \text { GLYALD }\end{array}$ & $2.7 \times 10^{-12} \cdot \exp (360 / \mathrm{T})$ \\
\hline $\mathrm{XO} 2+\mathrm{NO} 3$ & $\rightarrow$ & $\begin{array}{l}\mathrm{NO} 2+\mathrm{HO} 2+.25 \cdot \mathrm{CO}+.25 \cdot \mathrm{CH} 2 \mathrm{O}+.25 \cdot \text { GLYOXAL } \\
+.25 \cdot \mathrm{CH} 3 \mathrm{COCHO}+.25 \cdot \mathrm{HYAC}+.25 \cdot \text { GLYALD }\end{array}$ & $2.4 \times 10^{-12}$ \\
\hline $\mathrm{XO} 2+\mathrm{CH} 3 \mathrm{O} 2$ & $\rightarrow$ & $\begin{array}{l}.2 \cdot \mathrm{CH} 3 \mathrm{OH}+.8 \cdot \mathrm{HO} 2+.8 \cdot \mathrm{CH} 2 \mathrm{O}+.2 \cdot \mathrm{CO}+.1 \cdot \mathrm{GLYOXAL} \\
+.1 \cdot \mathrm{CH} 3 \mathrm{COCHO}+.1 \cdot \mathrm{HYAC}+.1 \cdot \mathrm{GLYALD}\end{array}$ & $5.0 \times 10^{-13} \cdot \exp (400 / \mathrm{T})$ \\
\hline $\mathrm{XO} 2+\mathrm{CH} 3 \mathrm{CO} 3$ & $\rightarrow$ & $\begin{array}{l}.25 \cdot \mathrm{CO}+.25 \cdot \mathrm{CH} 2 \mathrm{O}+.25 \cdot \mathrm{GLYOXAL}+\mathrm{CH} 3 \mathrm{O} 2+\mathrm{HO} 2 \\
+.25 \cdot \mathrm{CH} 3 \mathrm{COCHO}+.25 \cdot \mathrm{HYAC}+.25 \cdot \mathrm{GLYALD}+\{\mathrm{CO} 2\}\end{array}$ & $1.3 \times 10^{-12} \cdot \exp (640 / \mathrm{T})$ \\
\hline $\mathrm{OH}+\mathrm{BENZENE}$ & $\rightarrow$ & .53 PHENOL +.12 BEPOMUC $+.65 \mathrm{HO} 2+.35$ BENZO2 & $2.3 \times 10^{-12} \cdot \exp (-193 / \mathrm{T})$ \\
\hline $\mathrm{OH}+\mathrm{PHENOL}$ & $\rightarrow$ & $.14 \mathrm{PHENO} 2+.80 \mathrm{HO} 2+.06 \mathrm{PHENO}$ & $4.7 \times 10^{-13} \cdot \exp (1220 / \mathrm{T})$ \\
\hline $\mathrm{PHENO} 2+\mathrm{NO}$ & $\rightarrow$ & $\mathrm{HO} 2+.70$ GLYOXAL $+\mathrm{NO} 2$ & $2.6 \times 10^{-12} \cdot \exp (365 / \mathrm{T})$ \\
\hline $\mathrm{PHENO} 2+\mathrm{HO} 2$ & $\rightarrow$ & PHENOOH & $7.5 \times 10^{-13} \cdot \exp (700 / \mathrm{T})$ \\
\hline $\mathrm{OH}+\mathrm{PHENOOH}$ & $\rightarrow$ & PHENO2 & $3.8 \times 10^{-12} \cdot \exp (200 / \mathrm{T})$ \\
\hline $\mathrm{PHENO}+\mathrm{NO} 2$ & $\rightarrow$ & M & $2.1 \times 10^{-12}$ \\
\hline $\mathrm{PHENO}+\mathrm{O} 3$ & $\rightarrow$ & $\mathrm{C} 6 \mathrm{H} 5 \mathrm{O} 2$ & $2.8 \times 10^{-13}$ \\
\hline $\mathrm{C} 6 \mathrm{H} 5 \mathrm{O} 2+\mathrm{NO}$ & $\rightarrow$ & $\mathrm{PHENO}+\mathrm{NO} 2$ & $2.6 \times 10^{-2} \cdot \exp (365 / \mathrm{T})$ \\
\hline $\mathrm{C} 6 \mathrm{H} 5 \mathrm{O} 2+\mathrm{HO} 2$ & $\rightarrow$ & $\mathrm{C} 6 \mathrm{H} 5 \mathrm{OOH}$ & $7.5 \times 10^{-13} \cdot \exp (700 / \mathrm{T})$ \\
\hline $\mathrm{OH}+\mathrm{C} 6 \mathrm{H} 5 \mathrm{OOH}$ & $\rightarrow$ & $\mathrm{C} 6 \mathrm{H} 5 \mathrm{O} 2$ & $3.8 \times 10^{-12} \cdot \exp (200 / \mathrm{T})$ \\
\hline $\mathrm{BENZO} 2+\mathrm{NO}$ & $\rightarrow$ & $\mathrm{NO} 2+$ GLYOXAL +.5 BIGALD $1+\mathrm{HO} 2$ & $2.6 \times 10^{-12} \cdot \exp (365 / \mathrm{T})$ \\
\hline $\mathrm{BENZO} 2+\mathrm{HO} 2$ & $\rightarrow$ & BENZOOH & $7.5 \times 10^{-13} \cdot \exp (700 / \mathrm{T})$ \\
\hline $\mathrm{OH}+\mathrm{BENZOOH}$ & $\rightarrow$ & BENZO2 & $3.8 \times 10^{-12} \cdot \exp (200 / \mathrm{T})$ \\
\hline $\mathrm{MALO} 2+\mathrm{NO} 2$ & $\rightarrow$ & M & $\begin{array}{l}\mathrm{ko}=8.5 \mathrm{E}-29 \cdot(300 / \mathrm{T})^{6.5} \\
\mathrm{ki}=1.1 \times 10^{-11} \cdot(300 / \mathrm{T}) ; \mathrm{f}=0.6\end{array}$ \\
\hline $\mathrm{MALO} 2+\mathrm{NO}$ & $\rightarrow$ & $.4 \mathrm{GLYOXAL}+.4 \mathrm{HO} 2+.4 \mathrm{CO}$ & $7.5 \times 10^{-12} \cdot \exp (290 / \mathrm{T})$ \\
\hline $\mathrm{MALO} 2+\mathrm{HO} 2$ & $\rightarrow$ & $.16 \mathrm{GLYOXAL}+.16 \mathrm{HO} 2+.16 \mathrm{CO}$ & $4.3 \times 10^{-13} \cdot \exp (1040 / \mathrm{T})$ \\
\hline $\mathrm{OH}+\mathrm{TOLUENE}$ & $\rightarrow$ & $\begin{array}{l}.18 \mathrm{CRESOL}+.10 \mathrm{TEPOMUC}+.07 \mathrm{BZOO}+.65 \mathrm{TOLO} 2 \\
+.28 \mathrm{HO} 2\end{array}$ & $1.7 \times 10^{-12} \cdot \exp (352 / \mathrm{T})$ \\
\hline $\mathrm{OH}+\mathrm{CRESOL}$ & $\rightarrow$ & $.20 \mathrm{PHENO} 2+.73 \mathrm{HO} 2+.07 \mathrm{PHENO}$ & $4.7 \times 10^{-11}$ \\
\hline $\mathrm{BZOO}+\mathrm{HO} 2$ & $\rightarrow$ & $\mathrm{BZOOH}$ & $7.5 \times 10^{-13} \cdot \exp (700 / \mathrm{T})$ \\
\hline $\mathrm{OH}+\mathrm{BZOOH}$ & $\rightarrow$ & $\mathrm{BZOO}$ & $3.8 \times 10^{-12} \cdot \exp (200 / \mathrm{T})$ \\
\hline $\mathrm{BZOO}+\mathrm{NO}$ & $\rightarrow$ & $\mathrm{BZALD}+\mathrm{NO} 2+\mathrm{HO} 2$ & $2.6 \times 10^{-12} \cdot \exp (365 / \mathrm{T})$ \\
\hline $\mathrm{OH}+\mathrm{BZALD}$ & $\rightarrow$ & $\mathrm{ACBZO} 2$ & $5.9 \times 10^{-12} \cdot \exp (225 / \mathrm{T})$ \\
\hline $\mathrm{ACBZO} 2+\mathrm{NO} 2$ & $\rightarrow$ & PBZNIT & $\begin{array}{l}\mathrm{ko}=8.5 \mathrm{E}-29 \cdot(300 / \mathrm{T})^{6.5} \\
\mathrm{ki}=1.1 \times 10^{-11} \cdot(300 / \mathrm{T}) ; \mathrm{f}=0.6\end{array}$ \\
\hline PBZNIT & $\rightarrow$ & $\mathrm{ACBZO} 2+\mathrm{NO} 2$ & $\begin{array}{l}\mathrm{k}(\mathrm{ACBZO} 2+\mathrm{NO} 2) \\
\cdot 1.111 \mathrm{E} 28 \cdot \exp (-14000 / \mathrm{T})\end{array}$ \\
\hline $\mathrm{ACBZO} 2+\mathrm{NO}$ & $\rightarrow$ & $\mathrm{C} 6 \mathrm{H} 5 \mathrm{O} 2+\mathrm{NO} 2$ & $7.5 \times 10^{-12} \cdot \exp (290 / \mathrm{T})$ \\
\hline $\mathrm{ACBZO} 2+\mathrm{HO} 2$ & $\rightarrow$ & $.4 \mathrm{C} 6 \mathrm{H} 5 \mathrm{O} 2+.4 \mathrm{OH}$ & $4.3 \times 10^{-13} \cdot \exp (1040 / \mathrm{T})$ \\
\hline $\mathrm{TOLO} 2+\mathrm{NO}$ & $\rightarrow$ & $\begin{array}{l}\mathrm{NO} 2+.6 \text { GLYOXAL }+.4 \mathrm{CH} 3 \mathrm{COCHO}+\mathrm{HO} 2+.2 \text { BIGALD } 1 \\
+.2 \text { BIGALD } 2+.2 \text { BIGALD } 3\end{array}$ & $2.6 \times 10^{-12} \cdot \exp (365 / \mathrm{T})$ \\
\hline $\mathrm{DICARBO} 2+\mathrm{HO} 2$ & $\rightarrow$ & $.4 \mathrm{OH}+.07 \mathrm{HO} 2+.07 \mathrm{CH} 3 \mathrm{COCHO}+.07 \mathrm{CO}+.33 \mathrm{CH} 3 \mathrm{O} 2$ & $4.3 \times 10^{-13} \cdot \exp (1040 / \mathrm{T})$ \\
\hline $\mathrm{DICARBO} 2+\mathrm{NO}$ & $\rightarrow$ & $\mathrm{NO} 2+.17 \mathrm{HO} 2+.17 \mathrm{CH} 3 \mathrm{COCHO}+.17 \mathrm{CO}+.83 \mathrm{CH} 3 \mathrm{O} 2$ & $7.5 \times 10^{-12} \cdot \exp (290 / \mathrm{T})$ \\
\hline MDIALO2 + $\mathrm{HO} 2$ & $\rightarrow$ & $.4 \mathrm{OH}+.33 \mathrm{HO} 2+.07 \mathrm{CH} 3 \mathrm{COCHO}+.14 \mathrm{CO}+.07 \mathrm{CH} 3 \mathrm{O} 2+.07$ GLYOXAL & $4.3 \times 10^{-13} \cdot \exp (1040 / \mathrm{T})$ \\
\hline MDIALO2 + NO & $\rightarrow$ & $\begin{array}{l}\mathrm{NO} 2+.83 \mathrm{HO} 2+.17 \mathrm{CH} 3 \mathrm{COCHO}+.35 \mathrm{CO}+.17 \mathrm{CH} 3 \mathrm{O} 2 \\
+.17 \text { GLYOXAL }\end{array}$ & $7.5 \times 10^{-12} \cdot \exp (290 / \mathrm{T})$ \\
\hline $\mathrm{DICARBO} 2+\mathrm{NO} 2$ & $\rightarrow$ & M & $\begin{array}{l}\mathrm{ko}=8.5 \times 10^{-29} \cdot(300 / \mathrm{T})^{6.5} \\
\mathrm{ki}=1.1 \times 10^{-11} \cdot(300 / \mathrm{T}) ; \mathrm{f}=0.6\end{array}$ \\
\hline MDIALO2 + NO2 & $\rightarrow$ & M & $\begin{array}{l}\mathrm{ko}=8.5 \times 10^{-29} \cdot(300 / \mathrm{T})^{6.5} \\
\mathrm{ki}=1.1 \times 10^{-11} \cdot(300 / \mathrm{T}) ; \mathrm{f}=0.6\end{array}$ \\
\hline
\end{tabular}


Table 4. Continued.

\begin{tabular}{|c|c|c|c|}
\hline Reactants & & Products & Rate Constant(s) \\
\hline $\mathrm{OH}+\mathrm{XYLENES}$ & $\rightarrow$ & $\begin{array}{l}.15 \text { XYLOL }+.23 \text { TEPOMUC }+.06 \mathrm{BZOO}+.56 \text { XYLENO } 2 \\
+.38 \mathrm{HO} 2\end{array}$ & $1.7 \times 10^{-11}$ \\
\hline $\mathrm{OH}+\mathrm{XYLOL}$ & $\rightarrow$ & .30 XYLOLO $2+.63 \mathrm{HO} 2+.07$ PHENO & $8.4 \times 10^{-11}$ \\
\hline XYLOLO2 + NO & $\rightarrow$ & $\mathrm{HO} 2+\mathrm{NO} 2+.17$ GLYOXAL $+.51 \mathrm{CH} 3 \mathrm{COCHO}$ & $2.6 \times 10^{-12} \cdot \exp (365 / \mathrm{T})$ \\
\hline XYLOLO2 + HO2 & $\rightarrow$ & XYLOLOOH & $7.5 \times 10^{-13} \cdot \exp (700 / \mathrm{T})$ \\
\hline $\mathrm{OH}+\mathrm{XYLOLOOH}$ & $\rightarrow$ & XYLOLO2 & $3.8 \times 10^{-12} \cdot \exp (200 / \mathrm{T})$ \\
\hline XYLENO2 + HO2 & $\rightarrow$ & XYLENOOH & $7.5 \times 10^{-13} \cdot \exp (700 / \mathrm{T})$ \\
\hline $\mathrm{OH}+\mathrm{XYLENOOH}$ & $\rightarrow$ & XYLENO2 & $3.8 \times 10^{-12} \cdot \exp (200 / \mathrm{T})$ \\
\hline XYLENO2 + NO & $\rightarrow$ & $\begin{array}{l}\mathrm{NO} 2+\mathrm{HO} 2+.34 \text { GLYOXAL }+.54 \mathrm{CH} 3 \mathrm{COCHO} \\
+.06 \mathrm{BIGALD} 1+.2 \mathrm{BIGALD} 2+.15 \mathrm{BIGALD} 3+.21 \mathrm{BIGALD} 4\end{array}$ & $2.6 \times 10^{-12} \cdot \exp (365 / \mathrm{T})$ \\
\hline $\mathrm{MBO}+\mathrm{OH}$ & $\rightarrow$ & $\mathrm{MBOO} 2$ & $8.1 \times 10^{-12} \cdot \exp (610 / \mathrm{T})$ \\
\hline $\mathrm{MBO}+\mathrm{O} 3$ & $\rightarrow$ & $\begin{array}{l}.1 \mathrm{CO}+.5 \mathrm{CH} 2 \mathrm{O}+.1 \mathrm{CH} 3 \mathrm{COCH} 3+.9 \mathrm{HMPROP}+.25 \mathrm{HCOOH}+.25 \mathrm{CO} \\
+.06 \mathrm{HO} 2+.06 \mathrm{OH}\end{array}$ & $1.0 \times 10^{-17}$ \\
\hline $\mathrm{MBO}+\mathrm{NO} 3$ & $\rightarrow$ & $\mathrm{MBONO} 3 \mathrm{O} 2$ & $4.6 \times 10^{-14} \cdot \exp (-400 / \mathrm{T})$ \\
\hline $\mathrm{MBOO} 2+\mathrm{NO}$ & $\rightarrow$ & $\mathrm{HO} 2+.67 \mathrm{GLYALD}+.67 \mathrm{CH} 3 \mathrm{COCH} 3+.33 \mathrm{HMPROP}+.33 \mathrm{CH} 2 \mathrm{O}+\mathrm{NO} 2$ & $2.6 \times 10^{-12} \cdot \exp (365 / \mathrm{T})$ \\
\hline $\mathrm{MBOO} 2+\mathrm{CH} 3 \mathrm{O} 2$ & $\rightarrow$ & $\begin{array}{l}.917 \mathrm{CH} 2 \mathrm{O}+\mathrm{HO} 2+.25 \mathrm{CH} 3 \mathrm{OH}+.333 \mathrm{GLYALD}+.333 \mathrm{CH} 3 \mathrm{COCH} 3 \\
+.167 \mathrm{HMPROP}\end{array}$ & $3.75 \times 10^{-13} \cdot \exp (-40 / \mathrm{T})$ \\
\hline $\mathrm{MBOO} 2+\mathrm{HO} 2$ & $\rightarrow$ & MBOOOH & $7.5 \times 10^{-13} \cdot \exp (-700 / \mathrm{T})$ \\
\hline $\mathrm{MBONO} 3 \mathrm{O} 2+\mathrm{HO} 2$ & $\rightarrow$ & & $4.3 \times 10^{-13} \cdot \exp (1040 / \mathrm{T})$ \\
\hline $\mathrm{MBONO} 3 \mathrm{O} 2+\mathrm{NO}$ & $\rightarrow$ & $.25 \mathrm{HMPROP}+.25 \mathrm{CH} 2 \mathrm{O}+1.25 \mathrm{NO} 2+.75 \mathrm{ONIT}+.75 \mathrm{CH} 3 \mathrm{COCH} 3+.75 \mathrm{HO} 2$ & $2.6 \times 10^{-12} \cdot \exp (365 / \mathrm{T})$ \\
\hline $\mathrm{MBONO} 3 \mathrm{O} 2+\mathrm{NO} 3$ & $\rightarrow$ & $.25 \mathrm{HMPROP}+.25 \mathrm{CH} 2 \mathrm{O}+1.25 \mathrm{NO} 2+.75 \mathrm{ONIT}+.75 \mathrm{CH} 3 \mathrm{COCH} 3+.75 \mathrm{HO} 2$ & $2.4 \times 10^{-12}$ \\
\hline $\mathrm{MBOOOH}+\mathrm{OH}$ & $\rightarrow$ & $.5 \mathrm{MBOO} 2+.5 \mathrm{OH}$ & $3.8 \times 10^{-12} \cdot \exp (200 / \mathrm{T})$ \\
\hline $\mathrm{HMPROP}+\mathrm{OH}$ & $\rightarrow$ & HMPROPO2 & $1.4 \times 10^{-11}$ \\
\hline $\mathrm{HMPROPO} 2+\mathrm{NO}$ & $\rightarrow$ & $\mathrm{NO} 2+\mathrm{HO} 2+\mathrm{CH} 3 \mathrm{COCH} 3+\{\mathrm{CO} 2\}$ & $2.6 \times 10^{-12} \cdot \exp (365 / \mathrm{T})$ \\
\hline HMPROPO2 + HO2 & $\rightarrow$ & $.4 \mathrm{OH}+.4 \mathrm{HO} 2+.4 \mathrm{CH} 3 \mathrm{COCH} 3+\{.4 \cdot \mathrm{CO} 2\}$ & $4.3 \times 10^{-13} \cdot \exp (1040 / \mathrm{T})$ \\
\hline
\end{tabular}

Table 5. New photolysis reactions. ( $\mathrm{J}_{\mathrm{X}}$ is the photolysis rate for compound $X$ calculated by fTUV.)

\begin{tabular}{llll}
\hline Reactant & \multicolumn{2}{l}{ Products } & Rate \\
\hline $\mathrm{HONO}+\mathrm{h} v$ & $\rightarrow$ & $\mathrm{OH}+\mathrm{NO}$ & $\mathrm{J}_{\mathrm{HONO}}$ \\
$\mathrm{BIGALD} 1+\mathrm{h} v$ & $\rightarrow$ & $.6 \cdot \mathrm{MALO} 2+\mathrm{HO} 2$ & $0.140 \cdot \mathrm{J}_{\mathrm{NO}_{2}}$ \\
$\mathrm{BEPOMUC}+\mathrm{h} v$ & $\rightarrow$ & $\mathrm{BIGALD} 1+1.5 \cdot \mathrm{HO} 2+1.5 \cdot \mathrm{CO}$ & $0.100 \cdot \mathrm{J}_{\mathrm{NO}_{2}}$ \\
TEPOMUC $+\mathrm{h} v$ & $\rightarrow$ & $.5 \cdot \mathrm{CH} 3 \mathrm{CO} 3+\mathrm{HO} 2+1.5 \cdot \mathrm{CO}$ & $0.100 \cdot \mathrm{J}_{\mathrm{NO}_{2}}$ \\
BIGALD$+\mathrm{h} v$ & $\rightarrow$ & $.6 \cdot \mathrm{HO} 2+.6 \cdot \mathrm{DICARBO} 2$ & $0.200 \cdot \mathrm{J}_{\mathrm{NO}_{2}}$ \\
BIGALD3 $+\mathrm{h} v$ & $\rightarrow$ & $.6 \cdot \mathrm{HO} 2+.6 \cdot \mathrm{CO}+.6 \cdot \mathrm{MDIALO} 2$ & $0.200 \cdot \mathrm{J}_{\mathrm{NO}_{2}}$ \\
BIGALD$+\mathrm{h} v$ & $\rightarrow$ & $\mathrm{HO} 2+\mathrm{CO}+\mathrm{CH} 3 \mathrm{COCHO}+\mathrm{CH} 3 \mathrm{CO} 3$ & $0.006 \cdot \mathrm{J}_{\mathrm{NO}_{2}}$ \\
MBOOOH$+\mathrm{h} v$ & $\rightarrow$ & $\mathrm{OH}+\mathrm{HO} 2+.67 \cdot \mathrm{GLYALD}+.67 \cdot \mathrm{CH} 3 \mathrm{COCH} 3$ & $\mathrm{~J}_{\mathrm{CH}_{3} \mathrm{O}_{2} \mathrm{H}}$ \\
& & $+.33 \cdot \mathrm{HMPROP}+.33 \cdot \mathrm{CH} 2 \mathrm{O}$ & \\
$\mathrm{HMPROP}+\mathrm{h} v$ & $\rightarrow$ & $2 \cdot \mathrm{HO} 2+\mathrm{CO}+\mathrm{CH} 3 \mathrm{COCH} 3$ & $\mathrm{~J}_{\mathrm{GLYALD}}$ \\
\hline
\end{tabular}

laboratory studies (Kampf et al., 2013), including a substantial increase in effective Henry's law constant due to glyoxalsalt interactions. We have included all these proposed pathways into WRF-chem, and investigate their relative importance with simulations where only selected processes are active. Below we describe how each process is implemented.

Glyoxal can partition into the aerosol phase reversibly and irreversibly (Fig. 3):

(i) Reversible formation of monomer species (glyoxal, glyoxal monohydrate, and glyoxal dihydrate, pool 1 species) and oligomers (pool 2 species) are included based on Kampf et al. (2013), who observed an increase in the Henry's law constant by up to three orders of magnitude (compared to the value over pure water) in particles containing ammonium sulfate. This increase of the effective Henry's law constant, $K_{\mathrm{h} \text {, eq }}$, was attributed to salt-glyoxal interactions ("salting-

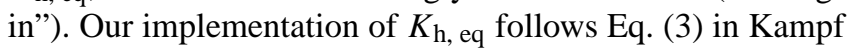
et al. (2013):

$K_{\mathrm{h}, \text { eq }}=K_{\mathrm{h}, \text { water }} / 10^{\left(-0.24 \min \left(12.0,\left(c_{\mathrm{as}}+c_{\mathrm{an}}\right)\right)\right)}$

where $K_{\mathrm{h} \text {, water }}=4.19 \times 10^{5} \mathrm{M} \mathrm{atm}^{-1}$ is the Henry's law constant of glyoxal over water, -0.24 the "salting-in" constant of ammonium sulfate, and $c_{\mathrm{as}}$ and $c_{\mathrm{an}}$ the concentrations $\left(\mathrm{mol} \mathrm{kg}^{-1}\right)$ of ammonium sulfate and nitrate respectively. 


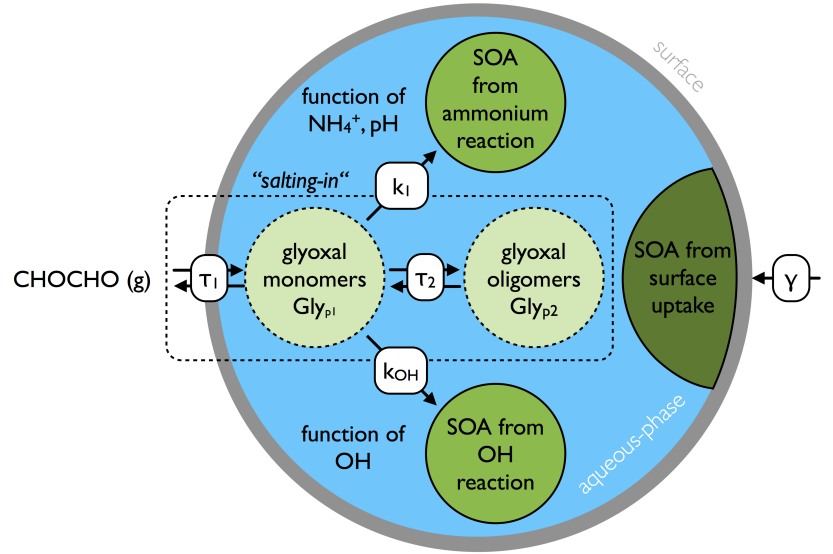

Figure 3. Schematic of pathways that can lead to SOA formation from glyoxal. $\gamma$ is the uptake coefficient of the surface reaction. $k_{I}$ the rate constant of the ammonium-catalyzed reaction, $k_{\mathrm{OH}}$ the one for the $\mathrm{OH}$ reaction. $\tau_{1}$ and $\tau_{2}$ are the characteristic time scales to fill the monomer/oligomer pool $\left(\mathrm{Gly}_{\mathrm{p} 1}\right.$ and Gly $\mathrm{p} 2$ respectively, as defined in Kampf et al., 2013). SOA in monomer and oligomer reservoirs is reversible. SOA in surface uptake, and ammonium and $\mathrm{OH}$ reaction reservoirs is irreversible.

Kampf et al. (2013) derived the "salting-in" constant for ammonium sulfate, constants for other seed particles are currently unmeasured. In this work we assume that ammonium nitrate acts like ammonium sulfate and their effects are additive. The sum $c_{\text {as }}+c_{\text {an }}$ in the calculation above is limited to $12 \mathrm{~mol} \mathrm{~kg}^{-1}$, per the results of Kampf et al. (2013), who found no further increase in $K_{\mathrm{h} \text {, eq }}$ at higher concentrations (of ammonium sulfate) within the time scales of their experiments.

The oligomerization constant $K_{\text {olig }}$ (as used by Ervens and Volkamer, 2010) defines the equilibrium partitioning between monomers (pool 1) and oligomers (pool 2) as

$\frac{\mathrm{Gly}_{\mathrm{p} 2, \mathrm{eq}}}{\mathrm{Gly}_{\mathrm{p} 1, \mathrm{eq}}}=K_{\mathrm{olig}}$,

with $\mathrm{Gly}_{\mathrm{p} 1 \text {,eq }}$ and $\mathrm{Gly}_{\mathrm{p} 2, \mathrm{eq}}$ the concentrations in each pool at equilibrium. Kampf et al. (2013) determined that $K_{\text {olig }}$ is approximately unity, for $c_{\mathrm{as}}<12 \mathrm{~mol} \mathrm{~kg}^{-1}$ and 0.5 for $c_{\text {as }}>12 \mathrm{~mol} \mathrm{~kg}^{-1}$. They also showed a significant timedependence of the rate at which the Henry's law equilibrium is established. In particular, the characteristic time scale for gas-particle partitioning of monomer species (glyoxal, monohydrate, and dihydrate) is on the order of minutes at $c_{\mathrm{as}}<12 \mathrm{~mol} \mathrm{~kg}^{-1}$, and several hours at $c_{\mathrm{as}}>12 \mathrm{~mol} \mathrm{~kg}^{-1}$. Both of these time scales are larger than a typical model time step in WRF-chem (30 s). To capture this time dependence in our model we calculate explicitly the glyoxal flux from the gas-phase to the monomer pool or vice versa as

$$
\frac{\partial \mathrm{Gly}_{\mathrm{p} 1}}{\partial t}=\frac{1}{\tau_{1}} \cdot\left(\mathrm{Gly}_{\mathrm{p} 1, \mathrm{eq}}-\mathrm{Gly}_{\mathrm{p} 1}\right)
$$

with Gly $_{\mathrm{p} 1}$ the current glyoxal monomer concentration in the aerosol liquid phase and $\tau_{1}$ the characteristic time scale for the monomer pool 1. Using the values derived in Kampf et al. (2013) for ammonium sulfate particles, we assume again that ammonium nitrate behaves like ammonium sulfate and use $\tau_{1}=2.5 \times 10^{2} \mathrm{~s}$ for $\left(c_{\mathrm{as}}+c_{\mathrm{an}}\right)<12 \mathrm{M}$, and $4.4 \times 10^{4} \mathrm{~s}$ for $\left(c_{\mathrm{as}}+c_{\mathrm{an}}\right)>12 \mathrm{M}$. Phase-separation of organic material with low $\mathrm{O} / \mathrm{C}$ (e.g., Bertram et al., 2011) was not considered in the calculation of the liquid-phase concentration.

The oligomer pool is in equilibrium with the monomer pool, with the flux defined similarly as

$\frac{\partial \mathrm{Gly}_{\mathrm{p} 2}}{\partial t}=\frac{1}{\tau_{2}} \cdot\left(\mathrm{Gly}_{\mathrm{p} 2, \mathrm{eq}}-\mathrm{Gly}_{\mathrm{p} 2}\right)$

with $\tau_{2}$ the characteristic time scale for the oligomer pool, and $\mathrm{Gly}_{\mathrm{p} 2}$ the current glyoxal concentration in the oligomer pool. In this work $\tau_{2}=5.5 \times 10^{3} \mathrm{~s}$ for $\left(c_{\mathrm{as}}+c_{\mathrm{an}}\right)$ $<12 \mathrm{~mol} \mathrm{~kg}^{-1}$, and $4.7 \times 10^{4} \mathrm{~s}$ for $\left(c_{\mathrm{as}}+c_{\mathrm{an}}\right)>12 \mathrm{~mol} \mathrm{~kg}^{-1}$ (based on values for ammonium sulfate in Kampf et al., 2013).

(ii) Three pathways for irreversible formation are considered: an ammonium-catalyzed volume pathway as presented in Noziere et al. (2009), an $\mathrm{OH}$ reaction volume pathway, and a surface uptake (both presented in Ervens and Volkamer, 2010).

The ammonium-catalyzed pathway is parameterized as a bulk (volume) reaction with a rate constant that depends exponentially on both particle acidity $(\mathrm{pH})$ and the activity of the ammonium ion $\left(a_{\mathrm{NH}_{4}}\right)$. Noziere et al. (2009) suggest a second-order rate constant from a two step reaction, but could not identify the second-order rate limiting step. We use this rate constant expression (Noziere et al., 2009, Eq. 12) as

$k_{\mathrm{II}}=2 \times 10^{-10} \cdot \exp \left(1.5 \cdot a_{\mathrm{NH}_{4}}\right) \cdot \exp (2.5 \cdot \mathrm{pH})$,

(in $\mathrm{M}^{-1} \mathrm{~s}^{-1}$ ) and convert into an apparent first-order rate constant $\left(k_{\mathrm{I}}\right)$ by multiplying with the particulate glyoxal concentration. We approximate $a_{\mathrm{NH}_{4}}$ as its concentration $\left(a_{\mathrm{NH}_{4}}\right.$ $=c_{\mathrm{NH}_{4}}$ ) because the thermodynamic module used (MESA Zaveri et al., 2005) does not provide activity coefficients for individual ions. The relationship between reversible and irreversible pathways is not well understood. On one hand, assuming that the ammonium-catalysis channel forms only irreversible products probably represents an upper limit estimate of the SOA formed from this volume process. On the other hand, reversibly formed oligomers do not evaporate easily; in fact the oligomer formation may even be accelerated as the concentration of monomer building blocks increases during the water evaporation process (De Haan et al., 2009). Furthermore, we assume that the total concentration in the monomer pool, which includes hydrated forms, is the only particulate glyoxal concentration available to the ammonium-catalyzed reaction. In practice also other forms of reversibly formed oligomers may be available for the ammonium-catalyzed reaction. 
Table 6. List of simulations conducted.

\begin{tabular}{ll}
\hline BASE & Reference simulation where glyoxal does not interact with particles. \\
RAW & Like BASE, but using the unmodified emission inventories. \\
SIMPLE & $\begin{array}{l}\text { Only surface uptake, active independent of aerosol phase state or composition, with } \\
\gamma=3.3 \times 10^{-3} \text {. Comparable to global model simulations (e.g., Fu et al., 2008). }\end{array}$ \\
VOLUME & $\begin{array}{l}\text { Reversible partitioning, } \mathrm{OH} \text { and ammonium-catalyzed volume reactions. } \\
\text { Reversible partitioning, } \mathrm{OH} \text { and ammonium-catalyzed volume reactions, and } \\
\text { HYBRID }\end{array}$ \\
& $\begin{array}{l}\text { surface uptake, with } \gamma=1.0 \times 10^{-3} \text {. Also surface uptake considers phase state. } \\
\text { Comparable to Waxman et al. }(2013) .\end{array}$ \\
FAST & $\begin{array}{l}\text { Like HYBRID, but glyoxal partitions into the liquid-phase (monomers, pool 1) } \\
\text { instantaneously. }\end{array}$ \\
FAST_PH & $\begin{array}{l}\text { Like HYBRID, but keeping fast time scales for the oligomer pool at high salt } \\
\text { concentrations. Further the dependence of SOA formation of the ammonium- } \\
\text { catalyzed channel shifted by } \Delta \mathrm{pH}=-2 .\end{array}$ \\
&
\end{tabular}

The $\mathrm{OH}$ pathway is a simplified version of what has been developed in Ervens and Volkamer (2010). Liquid-phase $\mathrm{OH}$ is assumed to be in equilibrium with the gas phase with a Henry's law constant of $25 \mathrm{M} \mathrm{atm}^{-1}$. Glyoxal is converted irreversibly into SOA by reaction with $\mathrm{OH}$ with a rate constant $k_{\mathrm{OH}}=1.1 \times 10^{9} \mathrm{M}^{-1} \mathrm{~s}^{-1}$ (Buxton et al., 1997). This rate constant has been derived for dilute solutions (cloud droplets), and is suggested to be a lower limit for more concentrated solutions like particles (Ervens and Volkamer, 2010). Like the ammonium reaction, the $\mathrm{OH}$ reaction also uses the total glyoxal concentration in the monomer pool.

The third irreversible pathway describes a surface uptake as

$\frac{\partial \mathrm{Gly}_{g}}{\partial t}=0.25 \cdot \gamma \cdot A \cdot \omega \cdot \mathrm{Gly}_{g}$

where $\gamma=1.0 \times 10^{-3}$ is the uptake coefficient, $A$ the aerosol surface area concentration $\left(\mathrm{cm}^{2} \mathrm{~cm}^{-3}\right)$, and $\omega$ the mean gasphase velocity of glyoxal under current conditions $\left(\mathrm{cm} \mathrm{s}^{-1}\right)$.

This latter pathway was used in previous global (Fu et al., 2008; Stavrakou et al., 2009), regional (Li et al., 2013), and other model studies (Volkamer et al., 2007; Washenfelder et al., 2011; Waxman et al., 2013) for scenarios without explicit glyoxal gas-to-particle partitioning and particle-phase chemistry; $\gamma$ here represents the probability of an irreversible reactive uptake of glyoxal into the particle. There is evidence that the chemical composition of the seed aerosol could have considerable effect on the uptake coefficient (Liggio et al., 2005; Trainic et al., 2011), a fact that we did not include in our simulations due to the lack of an experimentally supported parameterization.

The system of equations is integrated numerically using a 4th-order Runge-Kutta method (Press, 1992) at each call to the chemistry subsystem in WRF-chem for each size bin in MOSAIC. Only size bins which MOSAIC considers mixedphase or completely deliquesced are considered, except for the simulation in which only consider surface uptake ("SIMPLE", see next paragraph). All four glyoxal-SOA reservoirs (two reversible pools, volume pathway and surface uptake) in each size bin are treated as prognostic variables and hence subject to all physical processes acting upon particles in WRF-chem.

\subsection{Comparison of the formation pathways for glyoxal-SOA over California}

We conducted a total of seven simulations over the domain of California listed in Table 6 to evaluate our model and investigate the effects of different pathways and their interactions. In RAW and BASE, glyoxal has no interactions with particles. The BASE simulation is used to evaluate model performance against measurement data. In the RAW simulation we use the unmodified emission inventories to show the effects of our updated inventory. The SIMPLE case emulates previous modeling studies like Volkamer et al. (2007); Fu et al. (2008); Washenfelder et al. (2011), or Li et al. (2013), in which SOA formation from glyoxal is parameterized as surface uptake, only depends on available aerosol surface area, and does not consider aerosol chemical composition. Here, we implemented it without consideration of the phase state of the aerosol to estimate an upper limit of SOA production from glyoxal. Notably, Trainic et al. (2011) observed that surface limited uptake was highest under conditions when only interfacial water was present, and reproduced surface uptake limited SOA formation rates reported in Liggio et al. (2005). The VOLUME case assumes that SOA is formed through volume pathways - rather than a surface process. In the HYBRID case, both volume and surface processes are active, and both require a mixed-phase or deliquesced aerosol. In both the VOLUME and HYBRID studies reversible partitioning is also considered, and the increase in the Henry's law constant due to "salting-in" (Kampf et al., 2013) potentially increases the glyoxal available in the aqueous phase. Two additional sensitivity studies were made: FAST assumes that the increase in Henry's law constant due to "saltingin" does not have a time dependence. Hence in FAST glyoxal in the monomer pool (pool 1) is assumed to be in instantaneous equilibrium with the gas phase according to the 
"salted-in" Henry's law constant. The behavior of glyoxal oligomers (pool 2) is unchanged. With FAST_PH we investigate how the kinetic limitation of glyoxal partitioning at high salt concentrations affects contributions from oligomers, and assess the potential contribution of glyoxal to SOA in an environment with reduced aerosol acidity. As described in the previous section, the characteristic time scales to fill the monomer and oligomer pools change for salt concentrations $>12 \mathrm{~mol} \mathrm{~kg}^{-1}$. This translates into a slower partitioning of glyoxal into the particle-phase, and consequently no further increase in glyoxal for the ammonium-catalyzed reaction pathway. FAST_PH is based on FAST, hence also here we assume instantaneous equilibration for pool 1 (monomers). In FAST_PH we additionally keep the fast characteristic time scales for pool 2 (oligomers) at high salt concentrations. We further calculate ammonium-catalyzed reaction rates at a pH increased by 2 over the actual value. The exponential dependence of the ammonium-catalyzed reaction in Eq. (5) suggests a much stronger contribution at higher $\mathrm{pH}$, and indeed this dependence is largely responsible to explain findings in Mexico City (Volkamer et al., 2007; Waxman et al., 2013 ), where up to $15 \%$ of SOA is formed from glyoxal. The aerosol phase state is calculated by the MOSAIC module at each time step. In all simulations, with the exception of SIMPLE, SOA formation from glyoxal is shut off if the aerosol is dry.

\section{Model evaluation}

In order to investigate the atmospheric fate of glyoxal, the model needs to accurately simulate meteorology (transport patterns, radiation), the gas-phase precursors of glyoxal (isoprene, aromatics, ethyne), the oxidation capacity of the atmosphere $(\mathrm{OH})$, photolysis, and ambient aerosol characteristics. We performed a simulation over the domain of California in which glyoxal does not interact with particles (BASE, Table 6) and compared this against a combination of long-term ground measurements (CARB network, http://www.arb.ca. gov/aqmis2/aqmis2.php), as well as a number of supersites with advanced instrumentation (Fig. 1), and flights of several instrumented research aircraft during the CARES/CalNex campaigns. The aerosol modeling test bed (Fast et al., 2011b) provided these measurements in a common format, and we used it to extract the corresponding model variables that enable the statistical and graphical evaluation of the simulations listed in Table 6. The mean fractional bias

MFB $=\frac{2}{N} \sum_{i=1}^{N}\left(\frac{\operatorname{model}_{i}-\mathrm{obs}_{i}}{\operatorname{model}_{i}+\mathrm{obs}_{i}}\right) \cdot 100$

in $\%$, with $N$ the number of hourly observations $\left(\mathrm{obs}_{i}\right)$ and model results $\left(\operatorname{model}_{i}\right)$, is used as quantitative metric for model comparison throughout the following section.

Previous studies showed that similar configurations of WRF as used in this study have good skills in reproduc- ing transport patterns (Fast et al., 2011a) and meteorology (Angevine et al., 2012) over California. Comparison against CARB station data allowed for a general overview of model performance over the whole domain of California for routinely measured quantities. Evaluation plots can be found in the supplement, which we summarize here. The mean fractional bias is given as quantitative measure in parentheses. We found no significant biases in temperature $(<1 \%)$, shortwave downwelling radiation $(-12 \%)$ and relative humidity $(-2 \%)$. The diurnal variability of modeled temperature and relative humidity is underestimated. The model tends to overestimate wind speed $(50 \%)$. The height of the planetary boundary layer (PBLH) was additionally evaluated against ceilometer data at the LA ground site, where we find that nighttime and morning hours PBLH is comparable to measurements, while the modeled heights during the afternoon are lower than the observed heights (Fig. 6). Biases in modeled concentrations and diurnal cycles of $\mathrm{O}_{3}(2 \%), \mathrm{NO}_{\mathrm{x}}$ $(10 \%), \mathrm{CO}(23 \%)$ and $\mathrm{SO}_{2}(59 \%)$ in the BASE simulation are reasonable, except for $\mathrm{SO}_{2}$. Morning and evening (rush hour) peaks observed in $\mathrm{NO}_{\mathrm{x}}$ are also found in the model results. A similar morning peak in observed $\mathrm{CO}$ concentrations is not represented in the model, probably due to a lack of such a peak in the $\mathrm{CO}$ emissions inventory. $\mathrm{PM}_{2.5}$ concentrations are overestimated (78\%), while $\mathrm{PM}_{10}$ is underestimated $(-17 \%)$, possibly indicating a wrong partitioning of emissions between different size bins. Dust and sea salt emissions are the most probable emission candidates responsible for these discrepancies.

All three main precursors to glyoxal, aerosol properties, and glyoxal itself were measured at least at one of the supersites (Fig. 1) during CARES/CalNex. In Figs. 4-6 we compare averaged diurnal cycles of measurements at the supersites (T0, T1, Bakersfield, Pasadena) against the results from our WRF-chem simulations, and show the improvements made through the updated emission inventory.

Gas-phase precursors were measured by gas chromatography-mass spectrometry techniques at all supersites. Benzene is overestimated at T1 and Pasadena and matches observations at T0 (Fig. 4). Note that this is the case despite the fact that we did not use the even higher benzene emissions from Borbon et al. (2013). Modeled toluene agrees with measurements in Pasadena and Bakersfield (MFB of -1 and $13 \%$ respectively), and is overestimated at $\mathrm{T} 1$ and underestimated at $\mathrm{T} 0$. The model overestimates xylenes in Pasadena (28\%) and Bakersfield (41\%) and again shows too low values at $\mathrm{T} 0(-47 \%) . \mathrm{C}_{2} \mathrm{H}_{2}$ (only measured at Pasadena) is above measurements during nighttime and exhibits a larger diurnal variability than the data, but there is significant improvement over the results with the original emission inventory. Isoprene at Pasadena shows a too early onset during morning hours, with a tendency to underestimate concentrations midday. There is a substantially high bias in isoprene concentrations at Bakersfield (86\%) which has been amplified by the changes we made to the emissions 

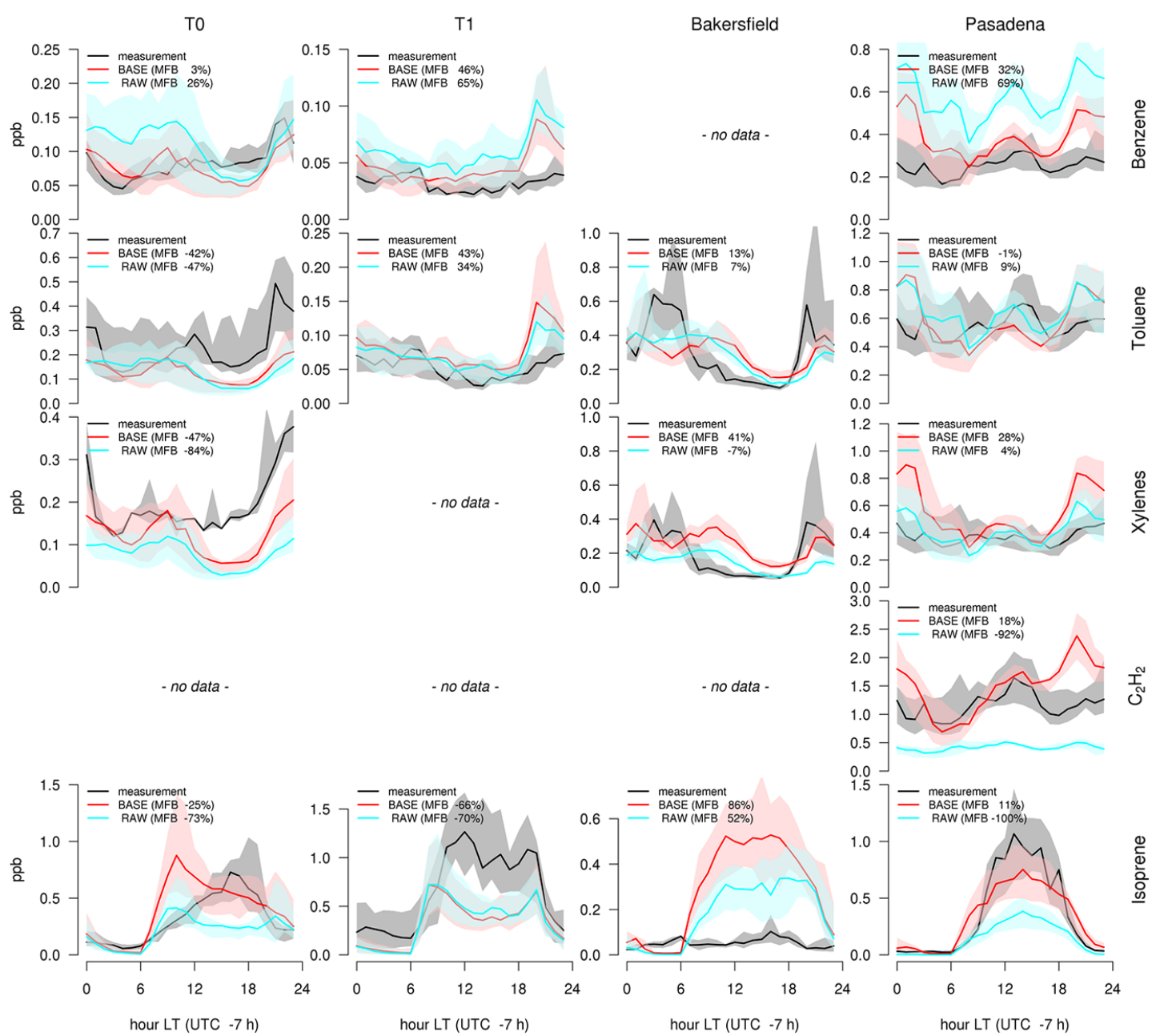

Figure 4. Averaged (29 May 00:00 UTC-16 June 00:00 UTC) diurnal cycles of measurements at the supersites (black) and model results using the original (RAW, blue) and updated emissions inventory (BASE, red) for the main precursors of glyoxal. Shown are the mean as solid line and the $25-75 \%$ range as shaded areas. MFB indicates mean fractional bias against measurements.

inventory. The diurnal cycle of isoprene is not captured at $\mathrm{T} 0$, but absolute values are reproduced, in contrast to $\mathrm{T} 1$, where the model correctly simulates the diurnal cycle, but concentrations are too low.

Measurements of aerosol chemical composition by the aerosol mass spectrometer (AMS) were available at all four supersites, and component analysis (positive matrix factorization, PMF) provided estimates of SOA concentrations at T1 (Setyan et al., 2012), Bakersfield (Liu et al., 2012b), and Pasadena (Hayes et al., 2013). In Fig. 5 we show a comparison against our model results. For this comparison the modeled size distribution is convolved with the AMS transmission function (see Knote et al. (2011) for a method description). While nitrate concentrations are comparable during the first half of the day, concentrations drop to zero at T0, $\mathrm{T} 1$ and Bakersfield sites - in contrast to observations. This is not observed at the Pasadena site. We suggest that this is not a major shortfall of the model, as the concentrations observed at T0, T1 and Bakersfield are low $\left(<0.2 \mu \mathrm{g} \mathrm{m}^{-3}\right)$, and even small errors in the calculations of thermodynamic equilibrium can lead to complete evaporation of nitrate in the model. There is a tendency of the model to overestimate aerosol sulfate except at Pasadena which we attribute to overestimated background concentration by the MOZART boundary conditions. Ammonium concentrations seem reasonable, if not somewhat too low in Pasadena. The model represents the diurnal evolution and absolute amounts of organic aerosol (OA) concentrations reasonable at T0, T1 and Bakersfield, while exhibiting a slight underestimation at Pasadena. Evaluation of SOA concentrations against PMF OOA factors at T1, Bakersfield, and Pasadena shows stronger underestimations than total OA, indicating that the simplified parameterization of Hodzic and Jimenez (2011) with the reduced yields is insufficient to represent SOA at these locations. Overall we find good agreement, lending some credibility to results that depend on the chemical composition of ambient aerosol. 


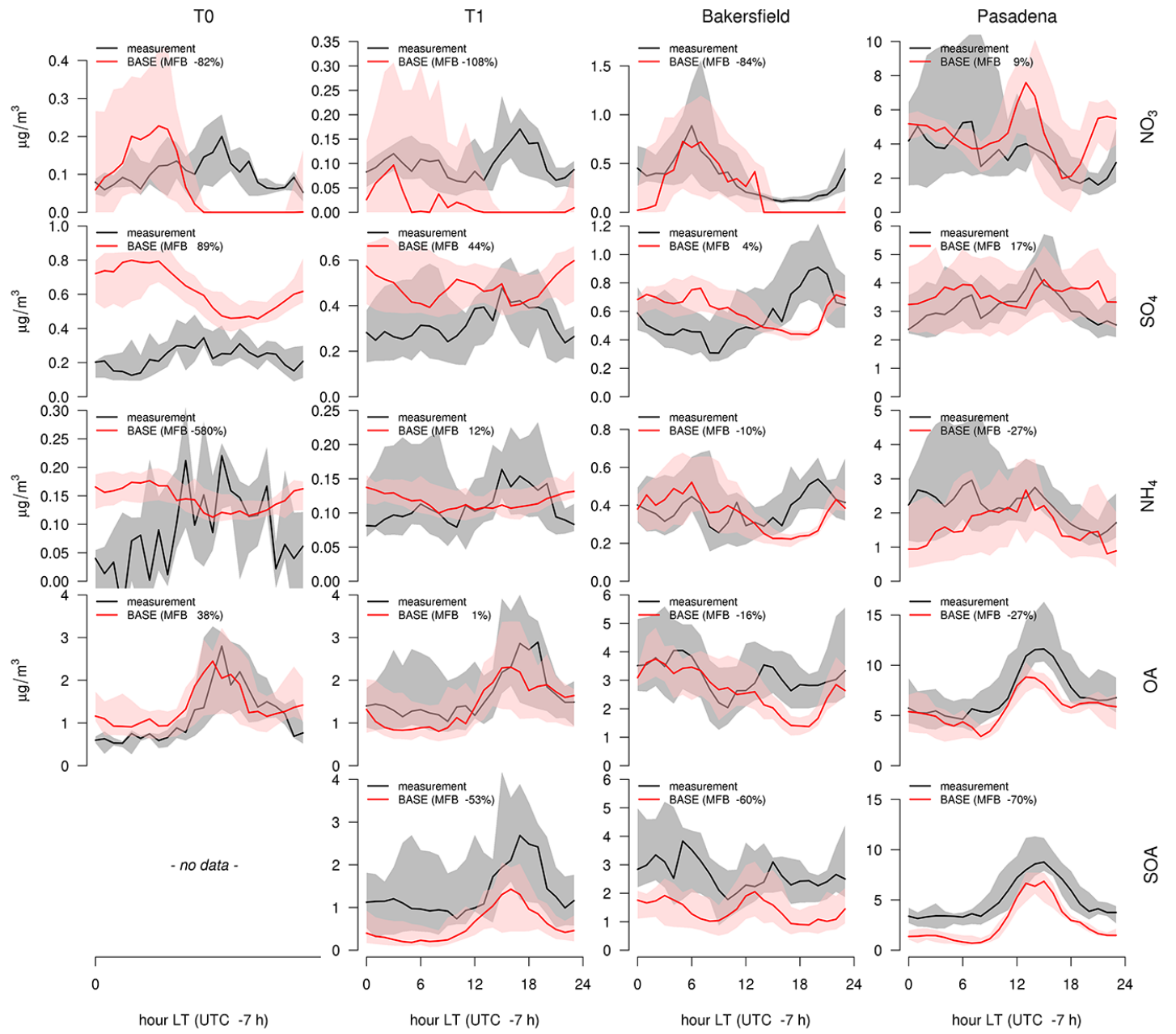

Figure 5. Averaged (29 May 00:00 UTC-16 June 00:00 UTC) diurnal cycles of model results (BASE, red) against aerosol mass spectrometer (AMS) measurements (black) at the four supersites for nitrate, sulfate, ammonium and organic sub-micron non-refractory aerosol mass. Lowest row shows comparison of modeled SOA against the sum of positive matrix factorization (PMF) factors MO-OOA plus LO-OOA (more and less oxidized oxygenated organic aerosol) at T1, and LVOOA plus SVOOA (low volatility and semivolatile oxygenated organic aerosol) at Bakersfield and Pasadena (see text for details). MFB indicates mean fractional bias against measurements.

The comparison of glyoxal against the two instruments stationed at the LA ground site (Fig. 6) shows that our simulations can capture the diurnal evolution, even though an underestimation of nighttime concentrations is found, and absolute values are slightly lower than observations. Note that there is no interaction between glyoxal and particles in the BASE/RAW simulations used in this comparison, meaning that the underestimation will likely be even larger once the aerosol sink is added. Panel 2 in Fig. 6 (formaldehyde) shows that the model captures the diurnal cycle of chemically similar compounds. Vertical profiles of glyoxal have been retrieved from AMAX-DOAS measurements onboard the NOAA Twin Otter (Baidar et al., 2013a). Only one profile was available during the period investigated (on 15 June for a low approach over Bakersfield), and a comparison is shown in Fig. 7. We find that the model underestimates observed glyoxal concentrations between $500 \mathrm{~m}$ a.g.l. and the surface, agrees within uncertainties with measurements between 500 and $2000 \mathrm{~m}$ a.g.1., and overestimates glyoxal above $2000 \mathrm{~m}$ a.g.1.. Direct measurements of glyoxal at the surface (not shown) agree with the AMAX-DOAS profile results. We interpret this result as follows: the local conditions at Bakersfield are not well reproduced by the model, while the regional conditions - represented by the values between 500 and $2000 \mathrm{~m}$ a.g.l. - are reasonably captured. The inability of the model to simulate local conditions may stem from strongly overestimated isoprene concentrations at Bakersfield (Fig. 4), which would lead to a suppression of $\mathrm{OH}$, and consequently to an underestimation of glyoxal concentrations. At least part of the overestimation above $2000 \mathrm{~m}$ a.g.l. may be due to glyoxal consumption by SOA 


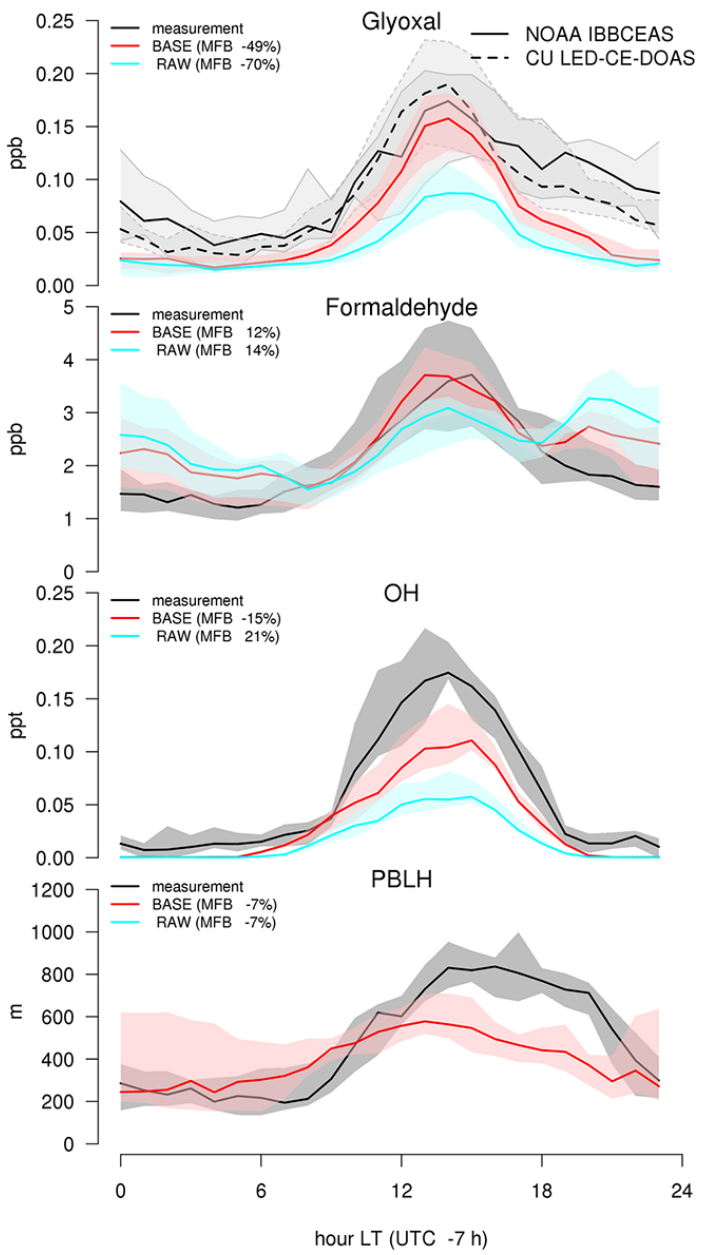

Figure 6. Averaged (29 May 00:00 UTC-16 June 00:00 UTC) diurnal cycles of glyoxal, formaldehyde, $\mathrm{OH}$ and boundary layer height (PBLH) at the LA ground site with model results using the original (RAW, blue) and updated emissions inventory (BASE, red). Glyoxal has been measured by two instruments at the LA ground site which are shown separately: Incoherent Broad Band Cavity Enhanced Absorption Spectroscopy (IBBCEAS) (Washenfelder et al., 2008) and University of Colorado at Boulder Light Emitting Diode Cavity Enhanced Differential Absorption Spectroscopy (CU LEDCE-DOAS) (Thalman and Volkamer, 2010).

formation within cloud water (refer e.g., to Carlton et al. (2008)), which we did not consider in this study.

The main sinks for glyoxal in the model are reaction with $\mathrm{OH}$, photolysis, and dry and wet deposition. $\mathrm{OH}$ concentrations (Fig. 6) are on average underestimated in the model when compared to measurements at the Pasadena ground site. While there is substantial uncertainty in how well models represent the hydroxyl radical, $\mathrm{OH}$ measurements have large uncertainties as well (Mao et al., 2012). Further, local conditions at the Pasadena ground site might not reflect the average concentrations over grid cell under consideration $(4 \times 4 \mathrm{~km})$. No significant biases have been found when com-

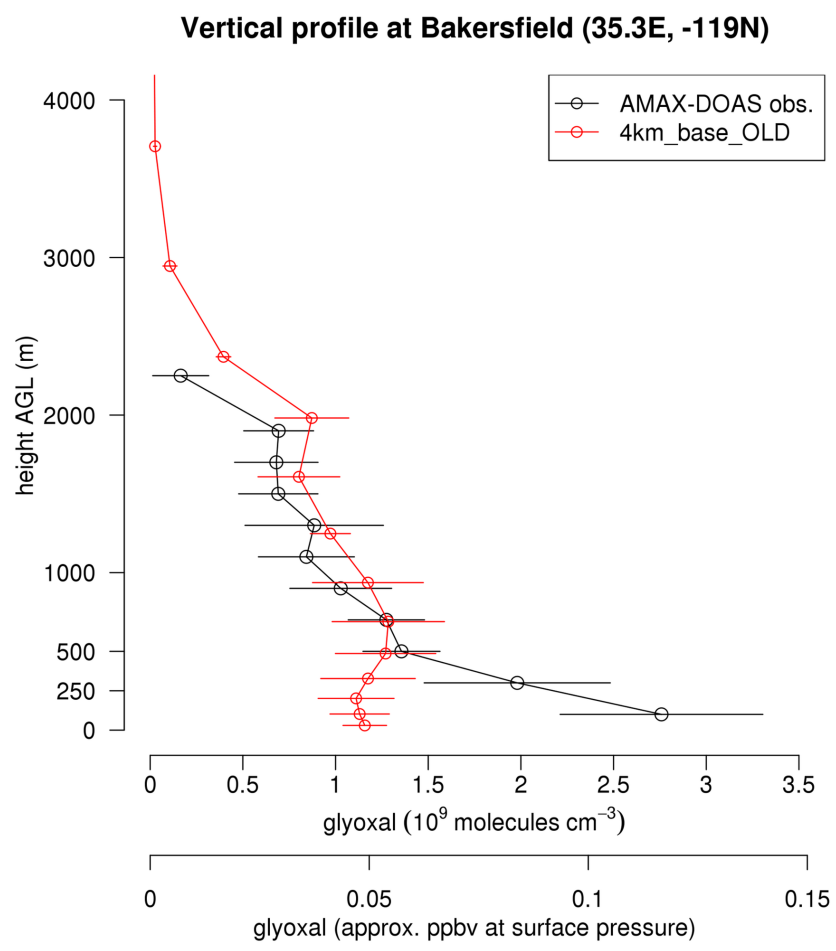

Figure 7. Comparison against vertical profiles of glyoxal as measured by AMAX-DOAS (black) on 15 June (low approach over Bakersfield (Baidar et al., 2013b) at about 21:25-21:40 UTC. Ascent and descent averaged $1 \sigma$ level errors). Model (red) error bars are 1 standard deviation of the 3 hourly values (11:00, 12:00, 13:00 LT).

paring photolysis rates of $\mathrm{O}\left({ }^{1} \mathrm{D}\right), \mathrm{NO}_{2}$, methylglyoxal and glyoxal against measurements onboard the NOAA WP-3D aircraft (see also Figure S3 in the supplement). There were no major precipitation events during the period of study, hence wet deposition is negligible (and not included in our simulations). We did not consider uptake and possible chemical reactions of glyoxal in cloud droplets.

\section{Regional variability of glyoxal and its SOA formation}

In the following we give a general overview of the regional variability of glyoxal and associated SOA formation over the California model domain, and focus on two regions with distinct characteristics (Fig. 1): the LA basin region as representative of the densely populated South Coast Air Basin, and the eastern slopes of the Central Valley, as more rural region with high isoprene emissions (Dreyfus et al., 2002). We discuss the relative importance of the different pathways in this region, before we present the results of the continental-scale simulation. 

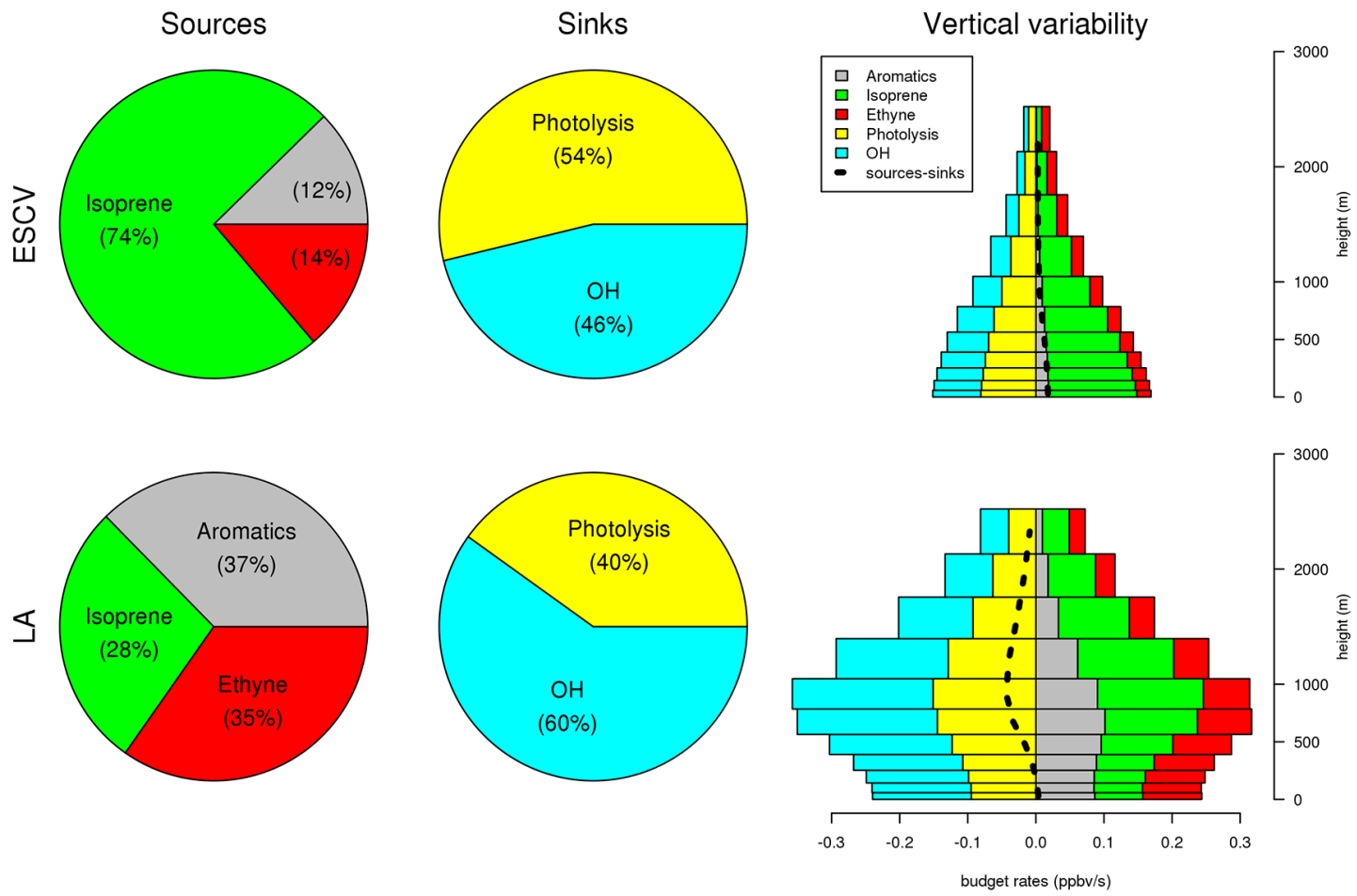

Figure 8. Relative importance of different photochemical sources and sinks of glyoxal in the LA basin and eastern slopes of the Central Valley focus region as time average over the simulation period in the lowest model level (pies), and its vertical variability (bar plot). Direct emissions of glyoxal were found to be minor $(<0.1 \%)$ and are not included in the plots. Results shown are from the BASE simulation where glyoxal does not interact with particles.

\subsection{The photochemical budget of glyoxal over California}

Photochemical source and sink strengths were tracked throughout the BASE model run (where glyoxal does not interact with particles) and used to derive mean budgets for the two focus regions (Fig. 8). Within the LA basin, aromatics $(37 \%)$, isoprene $(28 \%)$ and ethyne $(35 \%)$ contribute with comparable shares to glyoxal formation. This is in contrast to the findings of Washenfelder et al. (2011), where aromatics and isoprene contribute in total about $87 \%$ with equal shares, with ethyne only $11 \%$. This difference is likely due to the fact that we account for averaged regional contributions from the whole LA basin, compared to the budget at the Pasadena site itself as reported by Washenfelder et al. (2011). Pasadena is a rather residential neighborhood with more vegetation (i.e., isoprene) emissions and less strong emitters of aromatics and ethyne than other, more industrial and trafficintense areas in the LA basin. It is informative to compare this against a source budget over the isoprene-rich eastern slopes of the Central Valley. Here, isoprene gains importance as expected, now being responsible for three quarters of glyoxal formation (74\%).

$\mathrm{OH}$ and photolysis sinks both contribute substantially to total photochemical loss of glyoxal, with an equal share over the eastern slopes of the Central Valley, and $\mathrm{OH}$ being larger in the LA basin (60\%), due to strong pollutant sources and consequently higher radical concentrations. Washenfelder et al. (2011) find a comparable result in Pasadena, even though the photolysis sink is larger than the reaction with $\mathrm{OH}$ in their analysis, consistent with previous studies on local (Volkamer et al., 2007) and on global scales (Myriokefalitakis et al., 2008). Here again we suggest that these differences are likely due to the fact that we are analyzing an average air mass over the LA basin.

When analyzing the vertical variability of sources and sinks, we find a local maximum of both glyoxal production and removal rates between 500 and $1000 \mathrm{~m}$ a.g.l. (about the top of the boundary layer) in LA, which is not seen on the eastern slopes of the Central Valley. This is caused by higher concentrations of isoprene in these layers, probably the result of advection from regions with higher vegetation density. This hypothesis is further supported by the fact that net photochemistry is negative in these layers, indicating advection of glyoxal. The eastern slopes of the Central Valley were explicitly chosen as a region with high (local) isoprene emissions, hence ground level isoprene concentrations are the highest and the maximum is not seen aloft. These high local emissions of precursors also lead to a positive net photochemical budget near the ground in ESCV as advection moves glyoxal out of this area. 

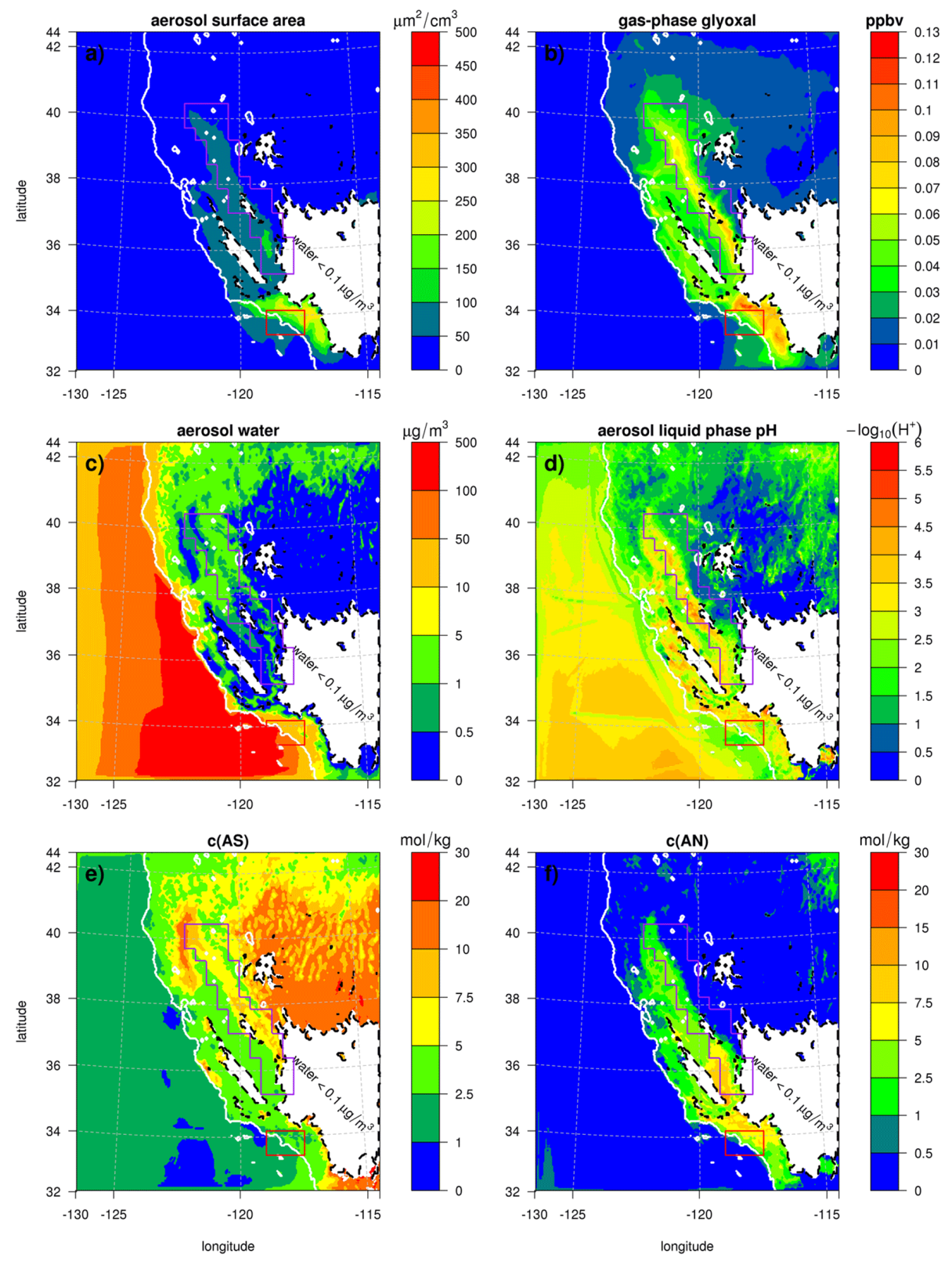

Figure 9. Maps of averaged aerosol related quantities in the lowest model layer (from top-left row-wise): (a) aerosol surface area, (b) gasphase glyoxal, (c) aerosol water, (d) aerosol pH, (e) ammonium sulfate and (f) ammonium nitrate salt concentrations in aerosol water. White lines delineate land mass. Regions with predominantly dry particles (avg. water content $<0.1 \mu \mathrm{g} \mathrm{m}^{-3}$ ) were masked. Salt concentrations and $\mathrm{pH}$ show mean for time steps where an aqueous aerosol phase existed. Red and purple outline indicate LA and ESCV focus regions respectively.

\subsection{Environmental parameters affecting SOA formation from glyoxal}

The panels in Fig. 9 give an overview of averaged quantities that affect the interactions of glyoxal with particles. Regions where the aerosol is usually dry (aerosol water content $<0.1 \mu \mathrm{g} \mathrm{m}^{-3}$ on average) are masked. As the glyoxalaerosol interactions most likely require a liquid-phase, no SOA formation occurs there in our simulations, except in the SIMPLE case. The LA basin is the hot spot for both aerosol surface area as well as gas-phase glyoxal. Additionally, the eastern slopes of the Central Valley (our second focus region) show elevated glyoxal concentration due to high availability of its precursors isoprene and MBO. Only slightly higher aerosol surface area concentrations are observed there. As expected, highest aerosol water concentrations are found over the oceans, but also several kilometers onshore. Only towards the northern part of the domain does 

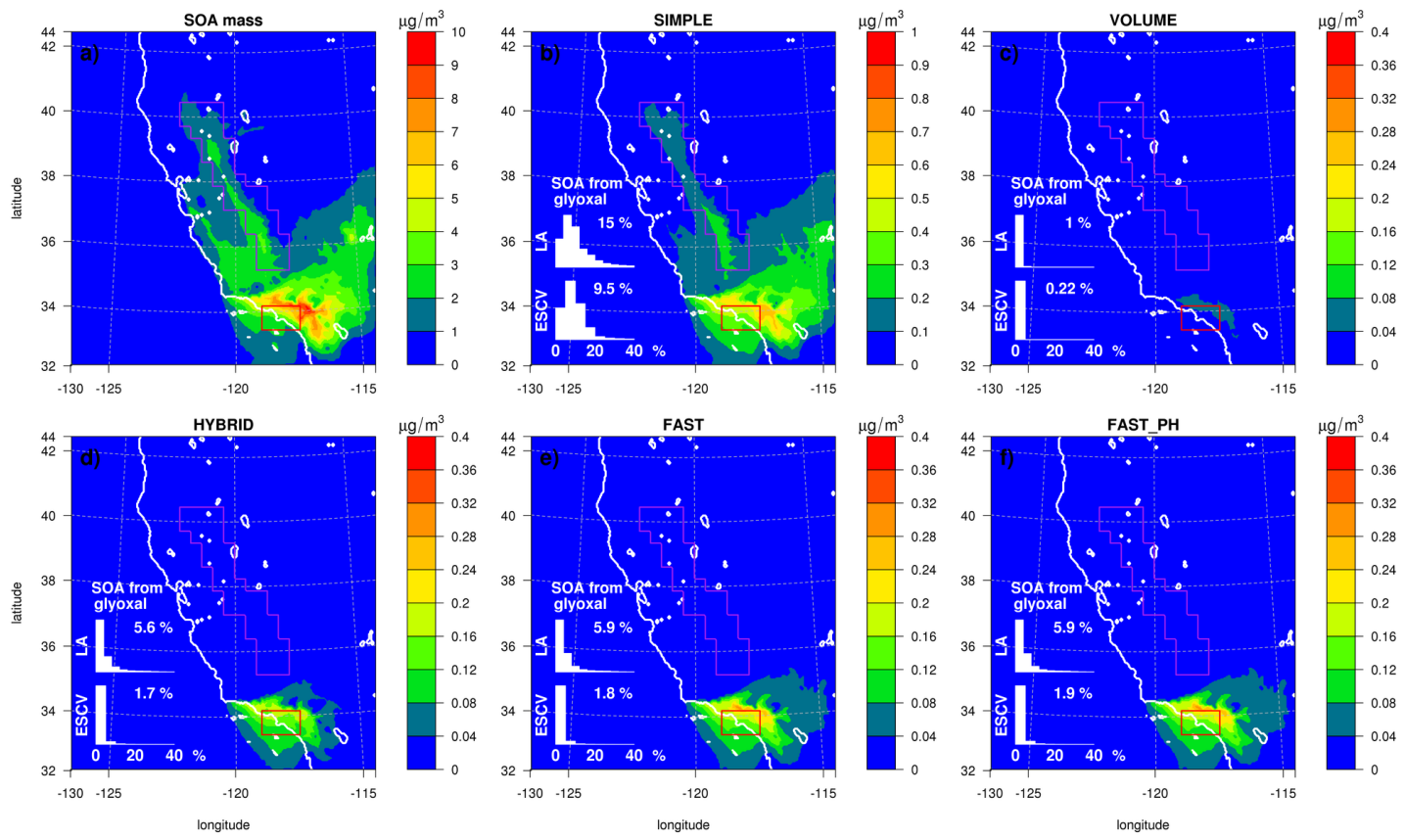

Figure 10. Average total SOA (BASE simulation, top left panel) and glyoxal-SOA masses (all other panels) in the lowest model vertical layer for the different studies. Note the differing scales. For each study, a histogram of the relative mass contribution of glyoxal to total SOA mass in the LA (red outline) and ESCV (purple) focus regions, as well as the average, is shown in the lower left corner of each panel. Histograms are based on the percent contribution of glyoxal SOA to total SOA for all grid points in the focus region at every hour of the simulation period.

the aerosol have substantial water concentrations further inland. Note the sharp contrast in aerosol water content between the LA basin and its surroundings. The $\mathrm{pH}$ of aerosol particles ranges between 1.5 and 5 and is typically around 3.5 , close to the values of $3.88 \pm 0.34$ (mean and std (standard deviation)) derived by explicit thermodynamic modeling of the conditions at the LA ground site presented in Liu et al. (2012a). Clearly visible is the $\mathrm{pH}$ lowering effect of emissions in the sea shipping lanes (e.g., parallel to $34.5^{\circ} \mathrm{N}$ ). Aerosol-phase ammonium salt concentrations are in general highest over land due to higher emissions and lower water concentrations. Ammonium sulfate and ammonium nitrate concentrations nonetheless exhibit very different spatial patterns. A stronger background contribution leads to a more dispersed pattern for sulfate concentrations, and we find the highest values over Nevada and the northern end of the Central Valley. Ammonium nitrate concentrations on the other hand are only substantial within the Central Valley and the LA basin. Note that we show salt concentration in the liquid phase of aerosol, which is not equivalent to absolute aerosol mass concentrations. For example, ammonium sulfate concentrations (in air) are comparably low over Nevada, but due to the low aerosol liquid water content in this region we find high values of the concentration in the aerosol water.

This combination of environmental factors makes coastal regions (extending several tens of kilometers from the shoreline) with strong biogenic and anthropogenic emissions very susceptible to formation of SOA from glyoxal, as all prerequisites (deliquesced aerosol, high surface area, high inorganic salt concentrations) are fulfilled in these regions. Note that while the surface uptake in HYBRID, FAST and FAST_PH currently only depends on the availability of a mixed-phase or deliquesced aerosol, both the reversible and ammonium-catalyzed pathway have additional dependencies on the chemical characteristics of the aerosol: (i) The ammonium-catalyzed reaction has an exponential dependence on the activity of the ammonium ion and aerosol pH (Noziere et al., 2009). (ii) The increase in the Henry's law constant for the reversible processes depends exponentially on the total concentration of ammonium sulfate in deliquesced particles, with an upper limit at $12 \mathrm{~mol} \mathrm{~kg}^{-1}$ (Kampf et al., 2013). A substantial area of the model domain is above this limit on average, especially if we assume (as done in this work) that ammonium nitrate has the same effect as ammonium sulfate and that their effects are additive. This indicates that "potential" for SOA formation from glyoxal is suppressed kinetically, possibly as a result of the increasing particle viscosity at very high salt concentrations.

\subsection{SOA formation from glyoxal over California}

A previous study using a surface uptake parameterization by Washenfelder et al. (2011) concluded that glyoxal would make a small contribution to SOA mass, between 

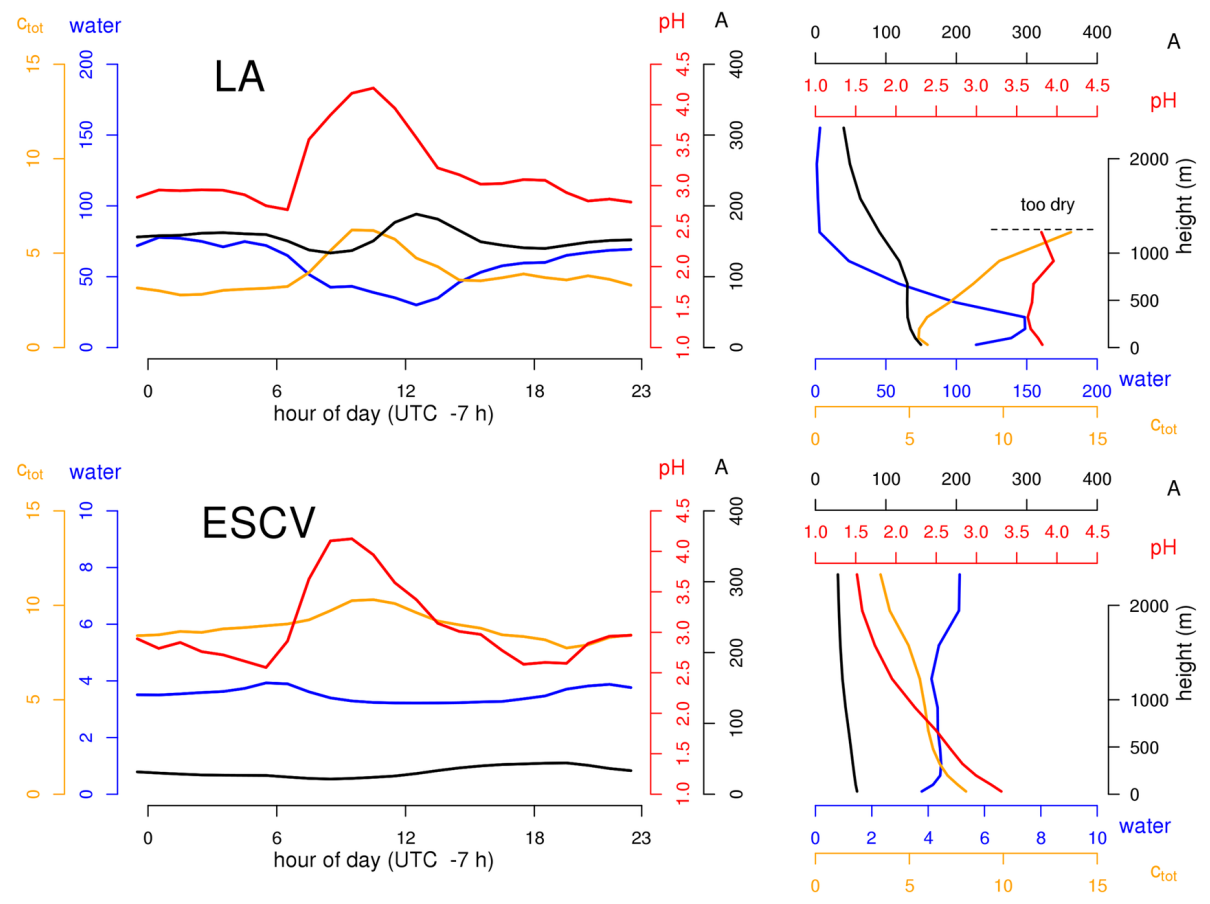

Figure 11. Averaged diurnal cycles (left panels) and vertical evolution (right) of environmental parameters affecting SOA formation from glyoxal in the two different focus regions: salt concentration in deliquesced particles $c_{\text {tot }}\left(\mathrm{mol} \mathrm{kg}^{-1}\right)$, aerosol water content $\left(\mu \mathrm{m}^{2} \mathrm{~cm}^{-3}\right)$, particle acidity $(\mathrm{pH})$, and aerosol surface area $\mathrm{A}\left(\mu \mathrm{m}^{2} \mathrm{~cm}^{-3}\right)$. Note different scales for water content between LA and ESCV. Values for $\mathrm{pH}$ and $c_{\text {tot }}$ masked above $1200 \mathrm{~m}$ a.g.l. in LA due to too few data points for statistically sound averaging.

$0-0.2 \mu \mathrm{g} \mathrm{m}^{-3}$ or 0 and $4 \%$, in California at the time period investigated. It is nonetheless instructive to look at the different total amounts and spatial patterns of SOA formed from glyoxal in the different sensitivity studies (Fig. 10). In the LA basin (BASE simulation) we find average SOA concentrations between 5 and $10 \mu \mathrm{g} \mathrm{m}^{-3}$. Depending on the pathways investigated, glyoxal contributes between $1.0 \%$ (VOLUME) and $15 \%$ (SIMPLE) of this SOA concentration. In the Central Valley, SOA mass is typically between 1 and $4 \mu \mathrm{g} \mathrm{m}^{-3}$, with highest values above the eastern slopes, and contributions from glyoxal between $0.22 \%$ (VOLUME) and $9.5 \%$ (SIMPLE).

\subsubsection{Spatial distribution}

All simulations show that the LA basin is the hot spot for SOA contribution from glyoxal over California, due to the high availability of precursors and existing aerosol. Most simulations also show elevated glyoxal-SOA concentrations over the eastern slopes of the Central Valley. In SIMPLE the highest amounts of glyoxal-SOA are formed, HYBRID, FAST and FAST_PH form about half the amounts of SIMPLE, and the VOLUME simulation a factor of 15 less.

In SIMPLE we do not consider aerosol phase state, which allows for constant SOA production as long as there is aerosol surface area available. All other simulations require the presence of a liquid-phase in the aerosol, including the surface uptake in HYBRID, FAST and FAST_PH. The VOLUME simulation shows that all volume processes if used as parameterized based on previous studies contribute about $1 \%$ of SOA in the LA basin $(0.22 \%$ in ESCV). Once we add a surface process (now dependent on a liquid-phase in the aerosol) about $5 \%$ (LA) of SOA are formed from glyoxal. Removing the kinetic limitation of gas-particle partitioning in FAST adds $0.3 \%$ in the LA basin and $0.1 \%$ in ESCV. Shifting the $\mathrm{pH}$ in FAST_PH adds an additional $0.1 \%$ of SOA in ESCV and less than $0.1 \%$ over LA.

\subsubsection{Diurnal evolution and vertical distribution}

In Fig. 11 we present diurnal cycles and vertical distribution for the relevant parameters affecting glyoxal-SOA production efficiency. Figure 12 shows the average diurnal cycle and vertical distribution of different glyoxal-SOA reservoirs in all four size bins, for all five simulations over the LA focus region. Figure 13 shows the same for the eastern slopes of the Central Valley (ESCV) focus region.

In both focus regions we find (Fig. 11) that average particle acidity is decreased at sunrise (increase in average particle $\mathrm{pH}$ to 4.0-4.5) which lasts until around noon, when acidity drops back to base levels ( $\mathrm{pH}$ of 3 ). We suggest that strong contributions from local, fresh emissions in the morning in both LA and ESCV lead to this observed increase in particle $\mathrm{pH}$. This is accompanied by an increase in salt concentrations 

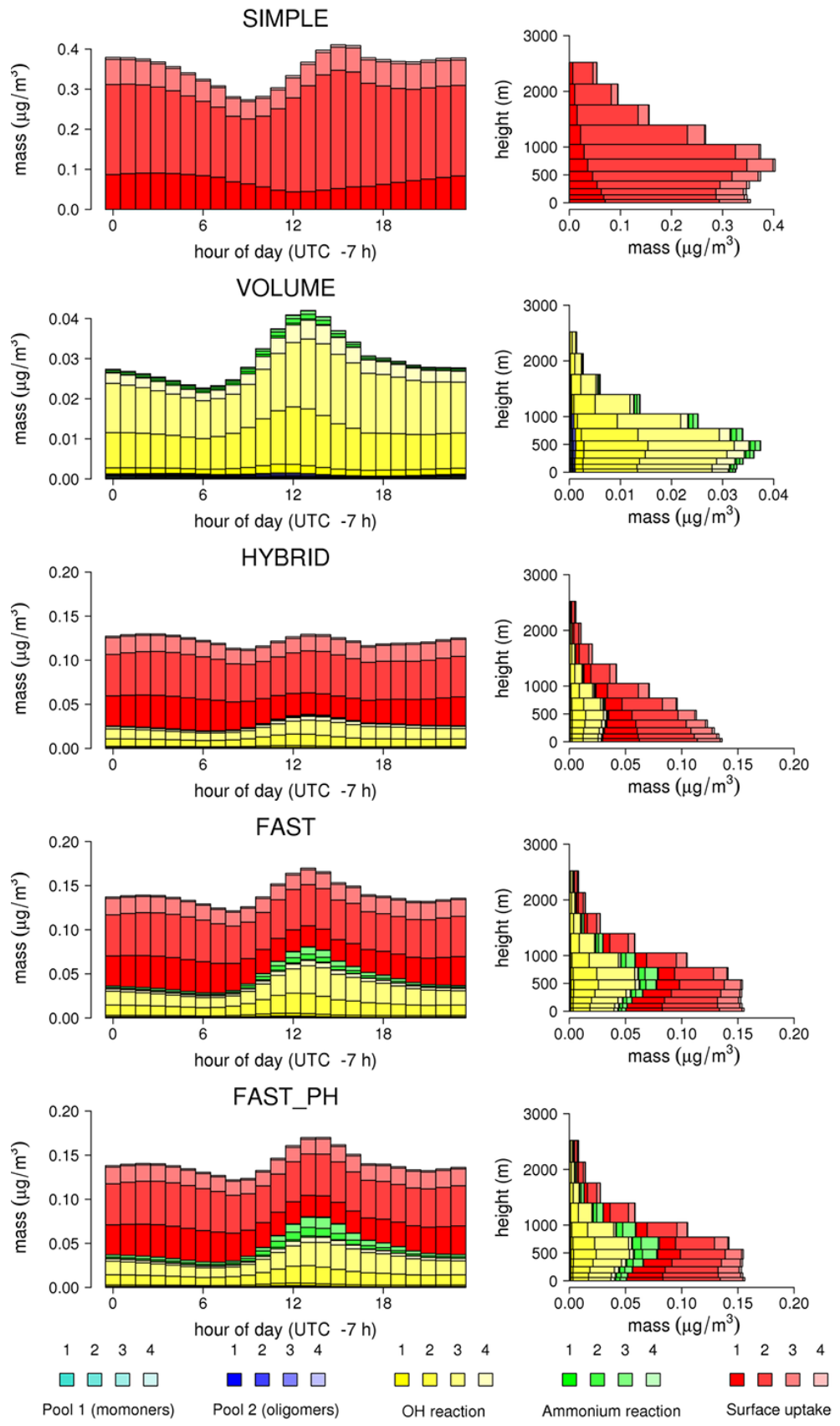

Figure 12. Contributions of different formation pathways to glyoxal-SOA in the LA basin focus region for the different studies given as diurnal cycle in the lowest model layer averaged over the simulation period (left panels), and as average vertical distribution (panels on the right). Size bins start with the smallest (1: $39-156 \mathrm{~nm}, 2: 156-625 \mathrm{~nm}, 3: 625 \mathrm{~nm}-2.5 \mu \mathrm{m}, 4: 2.5-10 \mu \mathrm{m})$. Note the different scales for each run.

and (in LA) decreasing aerosol water content. In ESCV the aerosol water content is very low, and remarkably constant throughout the day. The same holds true for aerosol surface area with average values of $30-50 \mu \mathrm{m}^{2} \mathrm{~cm}^{-3}$ in ESCV compared to $100-200 \mu^{2} \mathrm{~cm}^{-3}$ in LA. There, a diurnal cycle is visible with lower values in the morning and an early afternoon peak. Vertically, particles in the LA basin quickly dry out above $500 \mathrm{ma.g} .1$. and become almost completely dry above $1000 \mathrm{~m}$ a.g.l.. This is in sharp contrast to the findings for ESCV, where aerosol water content is even increasing with height. Here we also find decreasing $\mathrm{pH}$ values and salt concentrations with height.

In all simulations we find that (where applicable) the surface uptake and the $\mathrm{OH}$ pathway are the main contributors to 

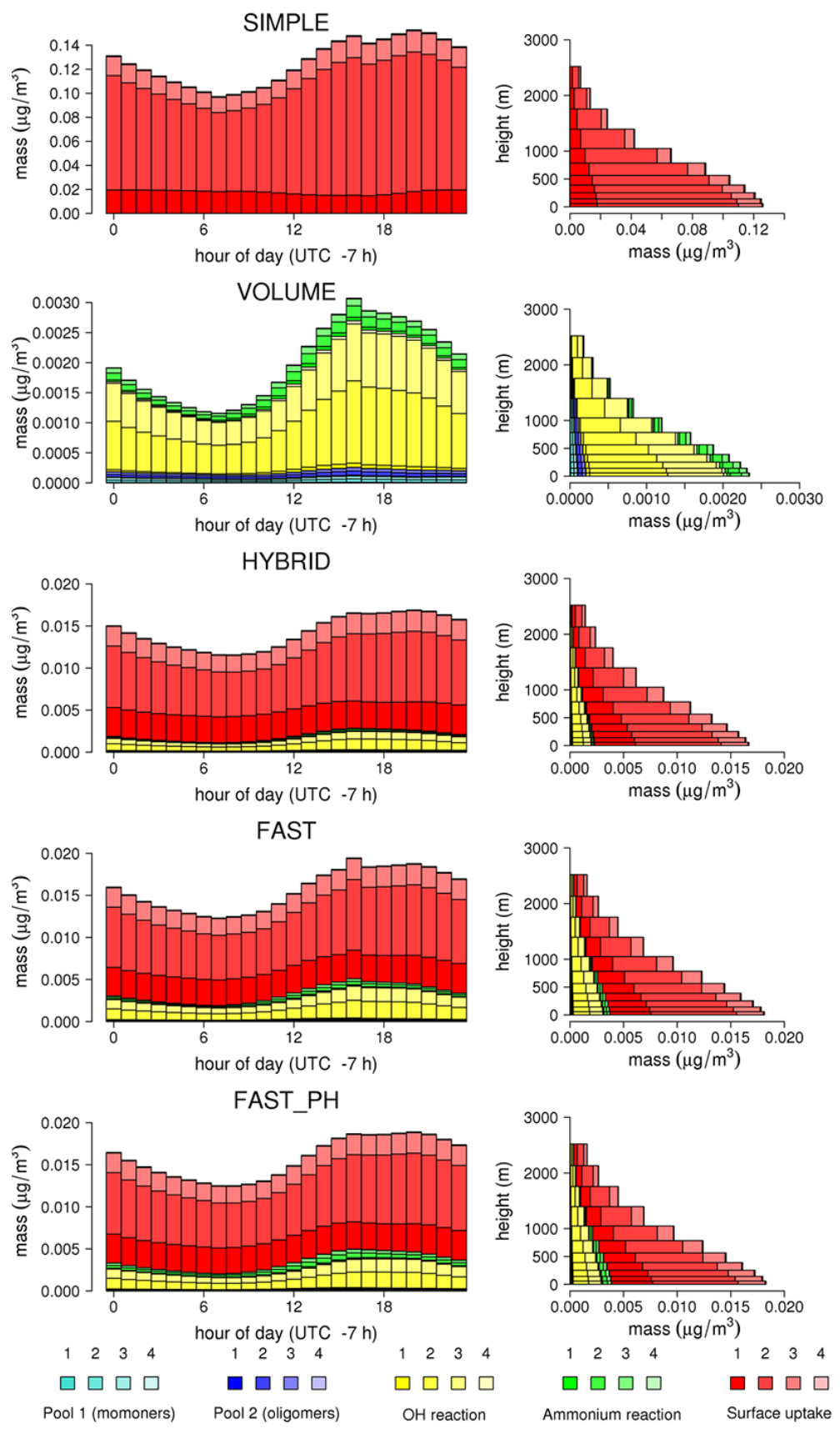

Figure 13. Like Fig. 12, but for the ESCV focus region. Note the different scales.

SOA formed from glyoxal (Figs. 12 and 13). In LA (Fig. 12) the simulations show a distinct noon peak in aerosol mass both from the surface uptake (corresponding to the peak in surface area) and the $\mathrm{OH}$ pathway (due to highest $\mathrm{OH}$ concentrations at noon, Fig. 6). The picture is different over the eastern slopes of the Central Valley (ESCV, Fig. 13). Here, the peak of glyoxal-SOA mass is shifted towards the evening without any of the governing environmental parameters showing a good correlation. This is a strong indication of different origins of glyoxal-SOA: locally produced (LA) versus advected (ESCV).

Comparison of the different formation pathways reveals that glyoxal SOA formed through the volume pathways alone (VOLUME) is a factor of 15 lower than from an irreversible reactive surface uptake (SIMPLE), even though the diurnal cycles are similar (Figs. 12 and 13). Within the volume pathways, $\mathrm{OH}$ reaction is the dominant source of SOA, with the ammonium pathway contributing about $5 \%$ (10\% in ESCV) and reversible partitioning $<5 \%$ mass. 
Once we combine a surface process with a volume process (HYBRID), now considering aerosol phase-state in the surface uptake, we form around $0.12 \mu \mathrm{g} \mathrm{m}^{-3} / 0.001 \mu \mathrm{g} \mathrm{m}^{-3}$ in LA/ESCV respectively. Around $80 \%$ (90\% in ESCV) of this mass is from the surface uptake. If we assume that gasparticle partitioning of glyoxal has no time dependence (instantaneous equilibration, FAST simulation), then the contribution of volume pathways increases notably, now forming about $40 \%$ of SOA from glyoxal in LA and $20 \%$ in ESCV. Still, the $\mathrm{OH}$ pathway is dominant. Finally, if we assume that there is no kinetic limitation at high salt concentrations and we pretend to be in an environment with less acidic particles (FAST_PH) we find that, while the overall contribution of volume pathways to glyoxal-SOA stays the same, between 10-20\% of this contribution is now from the ammonium pathway, and further we can identify $5-10 \%$ of mass as oligomers.

Looking at the vertical distribution we find in the ESCV focus region (Fig. 13) that concentrations almost linearly decrease with altitude, comparable to the vertical evolution of glyoxal turnover rates (Fig. 8). In LA we find that all simulations except HYBRID show a constant or even increasing glyoxal-SOA mass between 0 and $750 \mathrm{~m}$ a.g.l.. Notably, in FAST and FAST_PH the relative contribution of volume processes to total glyoxal SOA increases with height in these levels.

The FAST_PH simulation was a sensitivity study to investigate the partitioning behavior in regions where the aerosol $\mathrm{pH}$ is higher on average. In Mexico City the higher $\mathrm{pH}$ strongly increases the contribution of glyoxal to SOA (Waxman et al., 2013). However, we find only minor increases over FAST over California, where the limiting factor is not the ammonium-catalyzed reaction rate (Eq. 5), but the availability of glyoxal in the particle phase. This illustrates the strong impact that a kinetic limitation in reversible partitioning has on inhibiting the rate of glyoxal-SOA formation over California.

In terms of size distribution, the reversible and ammonium-catalyzed pathway put most glyoxal-SOA mass into bins $2(156-625 \mathrm{~nm})$ and $3(625 \mathrm{~nm}-2.5 \mu \mathrm{m})$, with the ammonium-catalyzed pathway favoring bin 3 . This is different for the surface uptake, which also adds substantial amount of mass to the smallest size bin (39-156 nm), and adds the majority into bin $2(156-625 \mathrm{~nm})$.

\subsubsection{Effects of the aerosol sink on gas-phase glyoxal}

To understand the impact of the aerosol sink for glyoxal on its gas-phase concentrations we show averaged absolute concentrations and differences against a simulation without aerosol sink (BASE) in Fig. 14.

In ESCV the effects of all parameterizations tested on gasphase glyoxal concentrations are negligible except for the SIMPLE case. Here, we find an average decrease of 5 pptv (or $10 \%$ of a 50 pptv daily average), with the largest differ-

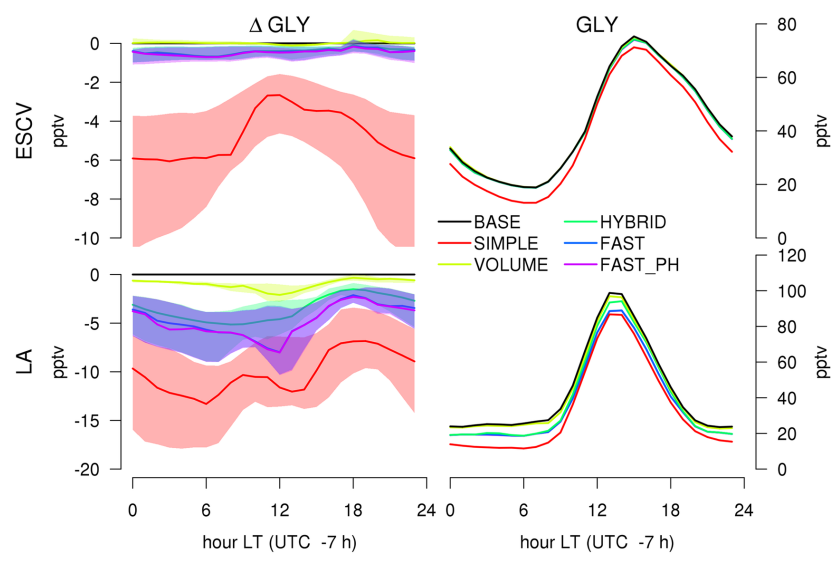

Figure 14. Changes in gas-phase glyoxal due to the different parameterizations of the aerosol sink relative to the BASE simulation. Shown are diurnal cycles for differences relative to the BASE simulation (left plots) and absolute concentrations (right plots) in the two focus regions, averaged over the simulation period. Shaded areas in differences represent the $25-75 \%$ value region.

ences during nighttime. In LA we find larger absolute differences $(-1$ pptv for VOLUME, around -5 pptv for HYBRID, FAST and FAST_PH, and -10 pptv for SIMPLE) which translate to relative changes up to $20 \%$ in absolute concentrations. Interestingly, and in contrast to what e.g., Washenfelder et al. (2011) show, we only see a clear daytime maximum in the differences against the BASE simulation if we look at the VOLUME, FAST or FAST_PH simulations. The surface process does not show a noon maximum, to the contrary, in ESCV it even has a distinct minimum at that time. If we now assume that the differences found in Washenfelder et al. (2011) are indeed attributable to an aerosol sink this would indicate that a volume process is more likely than a surface uptake.

No glyoxal is produced from gas-phase precursors at night in our simulations. However, glyoxal-SOA concentrations are sustained throughout the night in LA (Fig. 12) due to the still high levels of aerosol surface area on which available gas-phase glyoxal (Fig. 6) continues to be converted to SOA. In contrast to ESCV, where we see a clear downward trend in gas-phase glyoxal throughout the night (Fig. 14), concentrations in the LA basin remain constant throughout the night. This appears to be the result of direct emissions of glyoxal versus dilution and the aerosol sink. Interestingly, a depletion of gas-phase glyoxal was regularly observed during the morning rush-hour in Mexico City (see Fig. 2 in Volkamer et al., 2005) which we do not observe in LA. We note that particle surface area is much larger in Mexico City, and the associated time scales are shorter. The effect of fresh emissions on glyoxal uptake to aerosol deserves further investigation. 


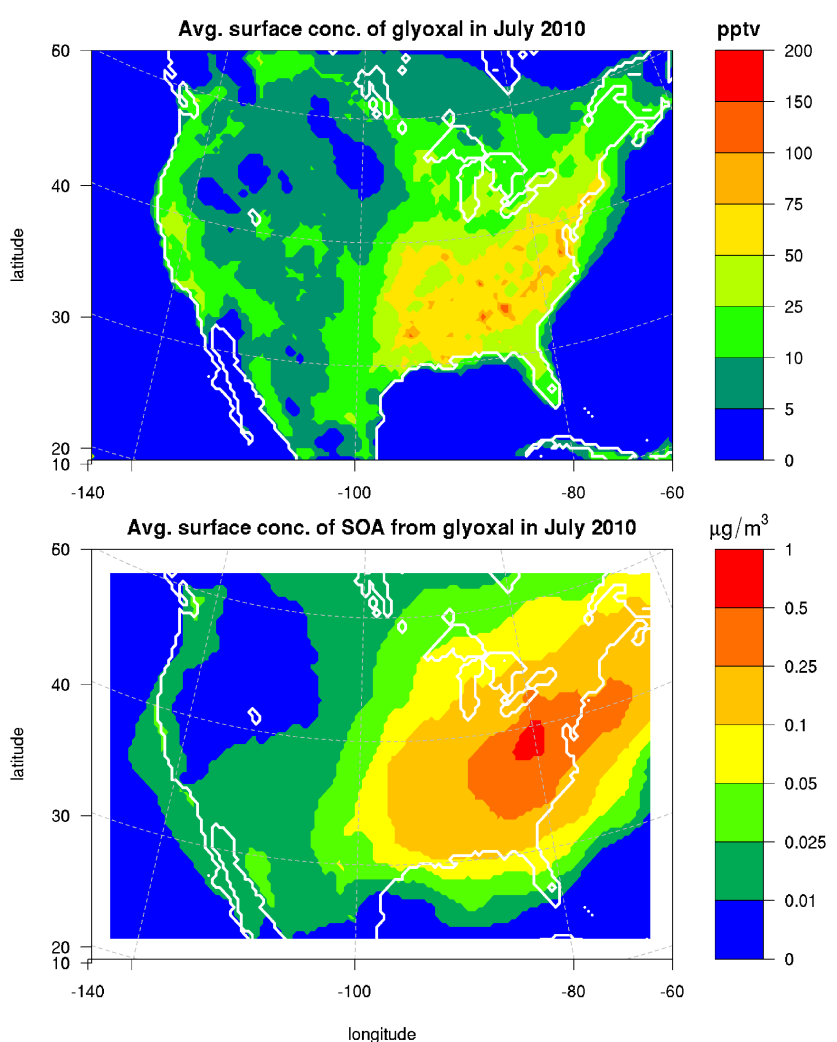

Figure 15. Average surface level concentration of gas-phase glyoxal (top) and SOA from glyoxal (bottom) in July 2010 over the continental United States. Simulation based on the VOLUME parameterization.

\subsection{SOA from glyoxal over the continental US}

We employed the model system as described above, using the VOLUME parameterization for glyoxal-SOA formation, to conduct a simulation over the continental US for the whole month of July 2010 . Thereby we could investigate the contribution of glyoxal to SOA at the continental scale. In Fig. 15 we show the average concentration of gas-phase glyoxal and SOA from glyoxal in the lowest model level. While glyoxalSOA concentrations over the western and central US are low, the eastern part of the US exhibits concentrations of up to $0.8 \mu \mathrm{g} \mathrm{m}^{-3}$ ( $15 \%$ of total SOA) with the highest values located over Ohio/Pennsylvania. Gas-phase glyoxal concentrations over the northeastern US are comparable to the ones over California, yet the northeastern US has higher concentrations of glyoxal-SOA. As we showed above, the gasparticle transfer of glyoxal (and hence SOA production from glyoxal) is hampered under dry conditions by a kinetic limitation in the increase in Henry's law constant. The comparison between the Northeast and California clearly shows this effect: while precursor concentrations are comparable, the amount of SOA produced from glyoxal is higher in the Northeast, as pollution levels there provide ample aerosol volume (and inorganic salts) to shift substantial amounts of glyoxal to the particle-phase while the humid conditions keep the salt concentrations in deliquesced particles below the levels where the kinetic limitation would come into play.

\section{Conclusions}

We presented a detailed investigation of plausible formation processes and the resulting regional variability of SOA from glyoxal using the WRF-chem model. Several parts of the model were extended to represent the glyoxal life cycle in more detail. We added several hypothetical mechanisms to form SOA from glyoxal: a very simple parameterization was compared with a number of more complex mechanisms. We considered surface as well as volume processes, and added reversible formation based on recent laboratory studies.

We then employed the model over the domain of California during the CARES/CalNex measurement campaigns to comprehensively evaluate its performance against measurements and to compare different ways to form SOA from glyoxal, as well as on a coarser resolution simulation over the continental United States to identify regions most susceptible to these SOA formation mechanisms.

Results from the simulations over California showed that the LA basin had the highest overall concentrations of SOA from glyoxal of the whole study domain in all simulations. A comparison of the simple surface uptake approach (SIMPLE) and ones considering aerosol phase state, volume and reversible processes (VOLUME, HYBRID) revealed that the simple approach leads to much higher concentrations (on average $0.5 \mu \mathrm{g} \mathrm{m}^{-3}$ or $15 \%$ of total SOA for SIMPLE, compared to $0.04 \mu \mathrm{g} \mathrm{m}^{-3}(1 \%)$ for VOLUME and $0.15 \mu \mathrm{g} \mathrm{m}^{-3}$ $(5.5 \%)$ for HYBRID). The phase-state constrained surface uptake in the more complex simulation (HYBRID) dominated with minor contributions $(<20 \%)$ from reversible formation and volume pathways. A kinetic limitation caps the increase in the Henry's law constant and reduces the time scales for its increase above the precipitation concentration of ammonium sulfate in aerosol water (phase transition). Once we remove this limitation for the gas-particle partitioning of glyoxal and assume instantaneous equilibration (FAST) the volume pathways contribute around $40 \%$ to glyoxal-SOA mass (20\% in ESCV). Further keeping a fast equilibration rate with the oligomer pool at high salt concentration leads to contributions up to $5 \%$ glyoxal-SOA mass from oligomers (FAST_PH). We find a minor further increase in glyoxal-SOA formed from the ammoniumcatalyzed pathway at the expense of the $\mathrm{OH}$ pathway once we artificially increase the aerosol $\mathrm{pH}$ handed over to the glyoxal-SOA module (FAST_PH). This increase is not exponential, as was expected from the exponential dependence of the rate constant. This is explained by the fact that, based on our current parameterization, the volume pathways are always limited by the availability of glyoxal in the 
liquid-phase, i.e., glyoxal does not reach steady state in particles due to viscosity limited mass transfer from the gas phase.

The SIMPLE simulation is based on chamber experiments and imbalance calculations, VOLUME only on laboratory results. HYBRID uses both, as do FAST and FAST_PH. The latter two simulations are pure sensitivity studies, as there is no laboratory evidence so far to remove the kinetic limitation, and no basis to increase the $\mathrm{pH}$ value. Measurements of the effect of salts other than ammonium sulfate on the Henry's law constant of glyoxal are currently not available and would be desirable to further our understanding of glyoxal-SOA formation. Given that VOLUME is based on explicitly identified pathways we consider it a lower limit on SOA production from glyoxal. As the surface uptake in SIMPLE does not depend on particle properties and is active as soon as surface area is available, we can consider it an upper limit.

The effect of the aerosol sink on average gas-phase glyoxal concentrations in the LA basin ranges between 0 and $15 \%$, with volume reactions (and not surface uptake) being the pathway able to reproduce a noon maximum difference in concentrations as previously found.

Results from the month-long $36 \mathrm{~km}$ resolution simulation over the domain of the continental United States revealed that the eastern, and especially the northeastern US, is more susceptible to SOA formation from glyoxal through volume pathways. This is despite comparable levels of gas-phase glyoxal are observed in California. We explain this by the fact that the formation of SOA from glyoxal is kinetically limited in the Western US due to dry conditions and high salt concentrations in the aerosol which hamper the gas-particle transfer of glyoxal.

\section{The Supplement related to this article is available online at doi:10.5194/acp-14-6213-2014-supplement.}

Acknowledgements. Ravan Ahmadov, Stu McKeen and Wayne Angevine (NOAA) were extremely helpful in deriving the new emissions inventory and setting up the whole modeling system. William C. Kuster, Jessica B. Gilman, Carsten Warneke and Joost de Gouw (NOAA) kindly provided VOC measurements taken at the Pasadena ground site during the CalNex campaign. F. Keutsch (UW Madison) kindly made measurements of glyoxal available in Bakersfield. B. Tom Jobson (WSU) provided VOC measurements at the T0 ground site. Aerosol mass spectrometer measurements at the T0 site were taken by Chen Song (PNNL). Philip S. Stevens (Indiana University) made $\mathrm{OH}$ measurements at Pasadena available. Lynn Russell and Shang Liu (UC San Diego) kindly provided positive matrix factorization (PMF) results for Bakersfield. We thank Barbara Ervens (NOAA), and also Siyuan Wang, and Christopher Kampf (CU Boulder) for fruitful discussions on glyoxal partitioning. Louisa Emmons is thanked for discussions regarding MOZART. This work has been funded by United States Department of Energy (US DOE) grant (BER, ASR program) DE-SC0006711. Jose-Luis Jimenez was also supported by CARB 11-305 and NSF AGS-1243354. Rainer Volkamer was supported by CARB 09-317, NSF CAREER award ATM-0847793, and US DOE contract DE-SC0006080. Jerome Fast was supported by the US NOAA Atmospheric Composition Climate Program (NA11OAR4310160) and the US DOE ASR program under Contract DE-AC06-76RLO 1830 at PNNL. The National Center for Atmospheric Research is sponsored by the National Science Foundation. Any opinions, findings and conclusions or recommendations expressed in the publication are those of the author(s) and do not necessarily reflect the views of the National Science Foundation.

Edited by: F. Keutsch

\section{References}

Ahlm, L., Liu, S., Day, D. A., Russell, L. M., Weber, R., Gentner, D. R., Goldstein, A. H., DiGangi, J. P., Henry, S. B., Keutsch, F. N., VandenBoer, T. C., Markovic, M. Z., Murphy, J. G., Ren, X., and Scheller, S.: Formation and growth of ultrafine particles from secondary sources in Bakersfield, California, J. Geophys. Res.-Atmos., 117, D00V08, doi:10.1029/2011JD017144, 2012.

Angevine, W. M., Eddington, L., Durkee, K., Fairall, C., Bianco, L., and Brioude, J.: Meteorological model evaluation for CalNex 2010, Mon. Weather Rev., 140, 3885-3906, 2012.

Baidar, S., Oetjen, H., Coburn, S., Dix, B., Ortega, I., Sinreich, R., and Volkamer, R.: The CU Airborne MAX-DOAS instrument: vertical profiling of aerosol extinction and trace gases, Atmos. Meas. Tech., 6, 719-739, doi:10.5194/amt-6-719-2013, 2013.

Baidar, S., Volkamer, R., Alvarez, R., Brewer, A., Davies, F., Langford, A., Oetjen, H., Pearson, G., Senff, C., and Hardesty, R. M. Combining Active and Passive Airborne Remote Sensing to Quantify $\mathrm{NO}_{2}$ and $\mathrm{O}_{x}$ Production near Bakersfield, CA, British Journal for Environmental and Climate Change, 3, 566-586, doi:10.9734/BJECC/2013/5740, 2013.

Bertram, A. K., Martin, S. T., Hanna, S. J., Smith, M. L., Bodsworth, A., Chen, Q., Kuwata, M., Liu, A., You, Y., and Zorn, S. R.: Predicting the relative humidities of liquid-liquid phase separation, efflorescence, and deliquescence of mixed particles of ammonium sulfate, organic material, and water using the organic-to-sulfate mass ratio of the particle and the oxygen-tocarbon elemental ratio of the organic component, Atmos. Chem. Phys., 11, 10995-11006, doi:10.5194/acp-11-10995-2011, 2011.

Bloss, C., Wagner, V., Jenkin, M. E., Volkamer, R., Bloss, W. J., Lee, J. D., Heard, D. E., Wirtz, K., Martin-Reviejo, M., Rea, G., Wenger, J. C., and Pilling, M. J.: Development of a detailed chemical mechanism (MCMv3.1) for the atmospheric oxidation of aromatic hydrocarbons, Atmos. Chem. Phys., 5, 641-664, doi:10.5194/acp-5-641-2005, 2005.

Borbon, A., Gilman, J., Kuster, W., Grand, N., Chevaillier, S., Colomb, A., Dolgorouky, C., Gros, V., Lopez, M., SardaEsteve, R., Holloway, J. S., Stutz, J., Petetin, H., McKeen, S., Beekman, M., Warneke, C., Parrish, D., and De Gouw, J.: Emission ratios of anthropogenic volatile organic compounds in northern mid-latitude megacities: observations versus emission inventories in Los Angeles and Paris, J. Geophys. Res.-Atmos., 118, 2041-2057, doi:10.1002/jgrd.50059, 2013. 
Brioude, J., Angevine, W. M., Ahmadov, R., Kim, S.-W., Evan, S., McKeen, S. A., Hsie, E.-Y., Frost, G. J., Neuman, J. A., Pollack, I. B., Peischl, J., Ryerson, T. B., Holloway, J., Brown, S. S., Nowak, J. B., Roberts, J. M., Wofsy, S. C., Santoni, G. W., Oda, T., and Trainer, M.: Top-down estimate of surface flux in the Los Angeles Basin using a mesoscale inverse modeling technique: assessing anthropogenic emissions of $\mathrm{CO}, \mathrm{NO}_{\mathrm{x}}$ and $\mathrm{CO}_{2}$ and their impacts, Atmos. Chem. Phys., 13, 3661-3677, doi:10.5194/acp-13-3661-2013, 2013.

Buxton, G. V., Malone, T. N., and Salmon, G. A.: Oxidation of glyoxal initiated by $\mathrm{OH}$ in oxygenated aqueous solution, J. Chem. Soc. Faraday, T., 93, 2889-2891, 1997.

Calvert, J. G., Atkinson, R., Becker, K. H., Kamens, R. M., Seinfeld, J. H., Wallington, T. J., and Yarwood, G.: The Mechanisms of Atmospheric Oxidation of Aromatic Hydrocarbons, Oxford University Press, New York, 1-540, 2002.

Carlton, A. G., Turpin, B. J., Altieri, K. E., Seitzinger, S., Reff, A., Lim, H.-J., and Ervens, B.: Atmospheric oxalic acid and SOA production from glyoxal: results of aqueous photooxidation experiments, Atmos. Environ., 41, 7588-7602, 2007.

Carlton, A. G., Turpin, B. J., Altieri, K. E., Seitzinger, S., Mathur, R., Roselle, S. J., and Weber, R. J.: CMAQ model performance enhanced when in-cloud secondary organic aerosol is included: Comparisons of organic carbon predictions with measurements, Environ. Sci. Technol., 42, 8798-8802, 2008.

Carter, W. P., Luo, D., and Malkina, I. L.: Documentation of the SAPRC-99 Chemical Mechanism for VOC Reactivity Assessment, California Environmental Protection Agency, Air Resources Board, Research Division, Riverside, California, 1-392, 2000.

Computational and Information Systems Laboratory. Yellowstone: IBM iDataPlex System (NCAR Community Computing). Boulder, CO: National Center for Atmospheric Research. http://n2t. net/ark:/85065/d7wd3xhc (last access: April 2014), 2012.

De Haan, D. O., Corrigan, A. L., Tolbert, M. A., Jimenez, J. L., Wood, S. E., and Turley, J. J.: Secondary organic aerosol formation by self-reactions of methylglyoxal and glyoxal in evaporating droplets, Environ. Sci. Technol., 43, 8184-8190, 2009.

Dreyfus, G. B., Schade, G. W., and Goldstein, A. H.: Observational constraints on the contribution of isoprene oxidation to ozone production on the western slope of the Sierra Nevada, California, J. Geophys. Res., 107, 4365, doi:10.1029/2001JD001490, 2002.

Duong, H. T., Sorooshian, A., Craven, J. S., Hersey, S. P., Metcalf, A. R., Zhang, X., Weber, R. J., Jonsson, H., Flagan, R. C., and Seinfeld, J. H.: Water-soluble organic aerosol in the Los Angeles Basin and outflow regions: airborne and ground measurements during the 2010 CalNex field campaign, J. Geophys. Res.Atmos., 116, D00V04, doi:10.1029/2011JD016674, 2011.

Emmons, L. K., Walters, S., Hess, P. G., Lamarque, J.-F., Pfister, G. G., Fillmore, D., Granier, C., Guenther, A., Kinnison, D., Laepple, T., Orlando, J., Tie, X., Tyndall, G., Wiedinmyer, C., Baughcum, S. L., and Kloster, S.: Description and evaluation of the Model for Ozone and Related chemical Tracers, version 4 (MOZART-4), Geosci. Model Dev., 3, 43-67, doi:10.5194/gmd3-43-2010, 2010.

Ervens, B. and Volkamer, R.: Glyoxal processing by aerosol multiphase chemistry: towards a kinetic modeling framework of secondary organic aerosol formation in aqueous particles, Atmos.
Chem. Phys., 10, 8219-8244, doi:10.5194/acp-10-8219-2010, 2010.

Ervens, B., Turpin, B. J., and Weber, R. J.: Secondary organic aerosol formation in cloud droplets and aqueous particles (aqSOA): a review of laboratory, field and model studies, Atmos. Chem. Phys., 11, 11069-11102, doi:10.5194/acp-1111069-2011, 2011.

Fast, J., Gustafson Jr, W., Easter, R., Zaveri, R., Barnard, J., Chapman, E., Grell, G., and Peckham, S.: Evolution of ozone, particulates, and aerosol direct radiative forcing in the vicinity of Houston using a fully coupled meteorologychemistry-aerosol model, J. Geophys. Res., 111, D21305, doi:10.1029/2005JD006721, 2006.

Fast, J. D., Gustafson Jr, W. I., Chapman, E. G., Easter, R. C., Rishel, J. P., Zaveri, R. A., Grell, G. A., and Barth, M. C.: The aerosol modeling testbed: a community tool to objectively evaluate aerosol process modules, B. Am. Meterol. Soc., 92, 343-360, 2011.

Fast, J. D., Gustafson Jr., W. I., Berg, L. K., Shaw, W. J., Pekour, M., Shrivastava, M., Barnard, J. C., Ferrare, R. A., Hostetler, C. A., Hair, J. A., Erickson, M., Jobson, B. T., Flowers, B., Dubey, M. K., Springston, S., Pierce, R. B., Dolislager, L., Pederson, J., and Zaveri, R. A.: Transport and mixing patterns over Central California during the carbonaceous aerosol and radiative effects study (CARES), Atmos. Chem. Phys., 12, 1759-1783, doi:10.5194/acp-12-1759-2012, 2012.

Fu, T.-M., Jacob, D. J., Wittrock, F., Burrows, J. P., Vrekoussis, M., and Henze, D. K.: Global budgets of atmospheric glyoxal and methylglyoxal, and implications for formation of secondary organic aerosols, J. Geophys. Res.-Atmos., 113, D15303, doi:10.1029/2007JD009505, 2008.

Galloway, M. M., Chhabra, P. S., Chan, A. W. H., Surratt, J. D., Flagan, R. C., Seinfeld, J. H., and Keutsch, F. N.: Glyoxal uptake on ammonium sulphate seed aerosol: reaction products and reversibility of uptake under dark and irradiated conditions, Atmos. Chem. Phys., 9, 3331-3345, doi:10.5194/acp-9-3331-2009, 2009.

Galloway, M. M., Huisman, A. J., Yee, L. D., Chan, A. W. H., Loza, C. L., Seinfeld, J. H., and Keutsch, F. N.: Yields of oxidized volatile organic compounds during the $\mathrm{OH}$ radical initiated oxidation of isoprene, methyl vinyl ketone, and methacrolein under high- $\mathrm{NO}_{\mathrm{x}}$ conditions, Atmos. Chem. Phys., 11, 10779-10790, doi:10.5194/acp-11-10779-2011, 2011.

Gentner, D. R., Isaacman, G., Worton, D. R., Chan, A. W., Dallmann, T. R., Davis, L., Liu, S., Day, D. A., Russell, L. M., Wilson, K. R., Gentner, D. R., Isaacman, G., Worton, D. R., Chan, A. W. H., Dallmann, T. R., Davis, L., Liu, S., Day, D. A., Russell, L. M., Wilson, K. R., Weber, R., Guha, A., Harley, R. A., and Goldstein, A. H.: Elucidating secondary organic aerosol from diesel and gasoline vehicles through detailed characterization of organic carbon emissions, P. Natl. Acad. Sci. USA, 109, 18318 $18323,2012$.

Goldstein, A. H. and Galbally, I. E.: Known and unexplored organic constituents in the earth's atmosphere, Environ. Sci. Technol., 41 1514-1521, 2007.

Grell, G., Peckham, S., Schmitz, R., McKeen, S., Frost, G., Skamarock, W., and Eder, B.: Fully coupled online chemistry within the WRF model, Atmos. Environ., 39, 6957-6975, 2005. 
Guenther, A., Karl, T., Harley, P., Wiedinmyer, C., Palmer, P. I., and Geron, C.: Estimates of global terrestrial isoprene emissions using MEGAN (Model of Emissions of Gases and Aerosols from Nature), Atmos. Chem. Phys., 6, 3181-3210, doi:10.5194/acp-63181-2006, 2006.

Hatakeyama, S., Washida, N., and Akimoto, H.: Rate constants and mechanisms for the reaction of hydroxyl (OD) radicals with acetylene, propyne, and 2-butyne in air at $297+/-2 \mathrm{~K}$, J. Phys. Chem., 90, 173-178, 1986.

Hayes, P., Ortega, A., Cubison, M., Froyd, K., Zhao, Y., Cliff, S., Hu, W., Toohey, D., Flynn, J., Lefer, B., Grossberg, N., Alvarez, S., Rappenglück, B., Taylor, J. W., Allan, J. D., Holloway, J. S., Gilman, J. B., Kuster, W. C., De Gouw, J., Massoli, P., Zhang, X., Liu, J., Weber, R. J., Corrigan, A. L., Russell, L. M., Isaacman, G., Worton, D. R., Kreisberg, N. M., Goldstein, A. H., Thalman, R., Waxman, E. M., Volkamer, R., Lin, Y. H., Surrat, J. D., Kleindienst, T. E., Offenberg, J. H., Dusanter, S., Griffith, S., Stevens, P. S., Brioude, J., Angevine, W. M., and Jimenez, J. L.: Organic aerosol composition and sources in Pasadena, California during the 2010 CalNex campaign, J. Geophys. Res.-Atmos., 118, 9233-9257, doi:10.1002/jgrd.50530, 2013.

Hersey, S. P., Craven, J. S., Metcalf, A. R., Lin, J., Lathem, T., Suski, K. J., Cahill, J. F., Duong, H. T., Sorooshian, A., Jonsson, H. H., Hersey, S. P., Craven, J. S., Metcalf, A. R., Lin, J., Lathem, T., Suski, K. J., Cahill, J. F., Duong, H. T., Sorooshian, A., Jonsson, H. H., Shiraiwa, M., Zuend, A., Nenes, A., Prather, K. A., Flagan, R. C., and Seinfeld, J. H.: Composition and hygroscopicity of the Los Angeles Aerosol: CalNex, J. Geophys. Res.-Atmos., 118, 3016-3036, doi:10.1002/jgrd.50307, 2013.

Hodzic, A. and Jimenez, J. L.: Modeling anthropogenically controlled secondary organic aerosols in a megacity: a simplified framework for global and climate models, Geosci. Model Dev., 4, 901-917, doi:10.5194/gmd-4-901-2011, 2011.

Holzinger, R., Goldstein, A. H., Hayes, P. L., Jimenez, J. L., and Timkovsky, J.: Chemical evolution of organic aerosol in Los Angeles during the CalNex 2010 study, Atmos. Chem. Phys., 13, 10125-10141, doi:10.5194/acp-13-10125-2013, 2013.

Kampf, C. J., Waxman, E. M., Slowik, J. G., Dommen, J., Pfaffenberger, L., Praplan, A. P., Prevot, A. S., Baltensperger, U., Hoffmann, T., and Volkamer, R.: Effective Henrys Law partitioning and the salting constant of glyoxal in aerosols containing sulfate, Environ. Sci. Technol., 47, 4236-4244, 2013.

Knote, C., Brunner, D., Vogel, H., Allan, J., Asmi, A., Äijälä, M., Carbone, S., van der Gon, H. D., Jimenez, J. L., KiendlerScharr, A., Mohr, C., Poulain, L., Prévôt, A. S. H., Swietlicki, E., and Vogel, B.: Towards an online-coupled chemistry-climate model: evaluation of trace gases and aerosols in COSMO-ART, Geosci. Model Dev., 4, 1077-1102, doi:10.5194/gmd-4-10772011, 2011.

Kroll, J. H., Ng, N. L., Murphy, S. M., Varutbangkul, V., Flagan, R. C., and Seinfeld, J. H.: Chamber studies of secondary organic aerosol growth by reactive uptake of simple carbonyl compounds. J. Geophys. Res.-Atmos., 110, D23207, doi:10.1029/2005JD006004, 2005.

Li, N., Fu, T.-M., Cao, J., Lee, S., Huang, X.-F., He, L.-Y., Ho, K.-F., Fu, J. S., and Lam, Y.-F.: Sources of secondary organic aerosols in the Pearl River Delta region in fall: Contributions from the aqueous reactive uptake of dicarbonyls, Atmos. Environ., 76, 200-207, 2013.

Liggio, J., Li, S., and McLaren, R.: Reactive uptake of glyoxal by particulate matter, J. Geophys. Res.-Atmos., 110, D10304, doi:10.1029/2004JD005113, 2005.

Lim, Y. B., Tan, Y., Perri, M. J., Seitzinger, S. P., and Turpin, B. J.: Aqueous chemistry and its role in secondary organic aerosol (SOA) formation, Atmos. Chem. Phys., 10, 10521-10539, doi:10.5194/acp-10-10521-2010, 2010.

Liu, J., Zhang, X., Parker, E. T., Veres, P. R., Roberts, J. M., Gouw, J. A., Hayes, P. L., Jimenez, J. L., Murphy, J. G., Ellis, R. A., Huey, L. G., and Weber, R. J.: On the gasparticle partitioning of soluble organic aerosol in two urban atmospheres with contrasting emissions: 2. Gas and particle phase formic acid, J. Geophys. Res.-Atmos., 117, D00V21, doi:10.1029/2012JD017912, 2012a.

Liu, S., Ahlm, L., Day, D. A., Russell, L. M., Zhao, Y., Gentner, D. R., Weber, R. J., Goldstein, A. H., Jaoui, M., Offenberg, J. H., Kleindienst, T. E., Rubitschun, C., Surrat, J. D., Sheesley, R. J., and Scheller, S.: Secondary organic aerosol formation from fossil fuel sources contribute majority of summertime organic mass at Bakersfield, J. Geophys. Res.-Atmos., 117, D00V26, doi:10.1029/2012JD018170, 2012b.

Mao, J., Ren, X., Zhang, L., Van Duin, D. M., Cohen, R. C., Park, J.H., Goldstein, A. H., Paulot, F., Beaver, M. R., Crounse, J. D., Wennberg, P. O., DiGangi, J. P., Henry, S. B., Keutsch, F. N., Park, C., Schade, G. W., Wolfe, G. M., Thornton, J. A., and Brune, W. H.: Insights into hydroxyl measurements and atmospheric oxidation in a California forest, Atmos. Chem. Phys., 12, 8009-8020, doi:10.5194/acp-12-8009-2012, 2012.

McDonald, B. C., Dallmann, T. R., Martin, E. W., and Harley, R. A.: Long-term trends in nitrogen oxide emissions from motor vehicles at national, state, and air basin scales, J. Geophys. Res.Atmos., 117, D00V18, doi:10.1029/2012JD018304, 2012.

McNeill, V. F., Woo, J. L., Kim, D. D., Schwier, A. N., Wannell, N. J., Sumner, A. J., and Barakat, J. M.: Aqueous-phase secondary organic aerosol and organosulfate formation in atmospheric aerosols: a modeling study, Environ. Sci. Technol., 46, 8075-8081, doi:10.1021/es3002986, 2012.

Myriokefalitakis, S., Vrekoussis, M., Tsigaridis, K., Wittrock, F., Richter, A., Brühl, C., Volkamer, R., Burrows, J. P., and Kanakidou, M.: The influence of natural and anthropogenic secondary sources on the glyoxal global distribution, Atmos. Chem. Phys., 8, 4965-4981, doi:10.5194/acp-8-4965-2008, 2008.

National Centers for Environmental Prediction/National Weather Service/NOAA/U.S. Department of Commerce, NCEP FNL Operational Model Global Tropospheric Analyses, continuing from July 1999, http://rda.ucar.edu/datasets/ds083.2, Research Data Archive at the National Center for Atmospheric Research, Computational and Information Systems Laboratory, Boulder, CO, updated daily, last access: 11 April 2013.

Nowak, J., Neuman, J., Bahreini, R., Middlebrook, A., Holloway, J., McKeen, S., Parrish, D., Ryerson, T., and Trainer, M.: Ammonia sources in the California South Coast Air Basin and their impact on ammonium nitrate formation, Geophys. Res. Lett., 39, L07804, doi:10.1029/2012GL051197, 2012.

Noziere, B., Dziedzic, P., and Cordova, A.: Products and kinetics of the liquid-phase reaction of glyoxal catalyzed by 
ammonium ions $\left(\mathrm{NH}_{4}^{+}\right)$, J. Phys. Chem. A, 113, 231-237, doi:10.1021/jp8078293, 2009.

O’Brien, R. E., Laskin, A., Laskin, J., Liu, S., Weber, R., Russell, L. M., and Goldstein, A. H.: Molecular characterization of organic aerosol using nanospray desorption/electrospray ionization mass spectrometry: CalNex 2010 field study, Atmos. Environ., 689, 265-272, doi:10.1016/j.atmosenv.2012.11.056, 2012.

Press, W. H.: Numerical Recipes in Fortran 77: The Art of Scientific Computing, vol. 1, Cambridge University Press, 1992.

Pusede, S. E. and Cohen, R. C.: On the observed response of ozone to $\mathrm{NO}_{\mathrm{x}}$ and VOC reactivity reductions in San Joaquin Valley California 1995-present, Atmos. Chem. Phys., 12, 8323-8339, doi:10.5194/acp-12-8323-2012, 2012.

Rollins, A., Browne, E., Min, K.-E., Pusede, S., Wooldridge, P., Gentner, D., Goldstein, A., Liu, S., Day, D., Russell, L., and Cohen, R. C.: Evidence for $\mathrm{NO}_{\mathrm{x}}$ control over nighttime SOA formation, Science, 337, 1210-1212, 2012.

Ryerson, T., Andrews, A., Angevine, W., Bates, T., Brock, C., Cairns, B., Cohen, R., Cooper, O., Gouw, J., Fehsenfeld, F., Ferrare, R. A., Fischer, M. L., Flagan, R. C., Goldstein, A. H., Hair, J. W., Hardesty, R. M., Hostetler, C. A., Jimenez, J. L., Langford, A. O., McCauley, E., McKeen, S. A., Molina, L. T., Nenes, A., Oltmans, S. J., Parrish, D. D., Pederson, J. R., Pierce, R. B., Prather, K., Quinn, P. K., Seinfeld, J. H., Senff, C. J., Sorooshian, A., Stutz, J., Surratt, J. D., Trainer, M., Volkamer, R., Williams, E. J., and Wofsy, S. C.: The 2010 California Research at the Nexus of Air Quality and Climate Change (CalNex) field study, J. Geophys. Res.-Atmos., 118, 5830-5866, doi:10.1002/jgrd.50331, 2013.

Sander, S. P., Abbatt, J., Barker, J. R., Burkholder, J. B., Friedl, R. R., Golden, D. M., Huie, R. E., Kolb, C. E., Kurylo, M. J., Moortgat, G. K., Orkin, V. L., and Wine, P. H.: Chemical kinetics and photochemical data for use in atmospheric studies, Evaluation No. 17, JPL Publication 10-6, Jet Propulsion Laboratory, Pasadena, available at: http://jpldataeval.jpl.nasa.gov (last access: July 2013), 2011.

Setyan, A., Zhang, Q., Merkel, M., Knighton, W. B., Sun, Y., Song, C., Shilling, J. E., Onasch, T. B., Herndon, S. C., Worsnop, D. R., Fast, J. D., Zaveri, R. A., Berg, L. K., Wiedensohler, A., Flowers, B. A., Dubey, M. K., and Subramanian, R.: Characterization of submicron particles influenced by mixed biogenic and anthropogenic emissions using high-resolution aerosol mass spectrometry: results from CARES, Atmos. Chem. Phys., 12, 8131-8156, doi:10.5194/acp-12-8131-2012, 2012.

Shilling, J. E., Zaveri, R. A., Fast, J. D., Kleinman, L., Alexander, M. L., Canagaratna, M. R., Fortner, E., Hubbe, J. M., Jayne, J. T., Sedlacek, A., Setyan, A., Springston, S., Worsnop, D. R., and Zhang, Q.: Enhanced SOA formation from mixed anthropogenic and biogenic emissions during the CARES campaign, Atmos. Chem. Phys., 13, 2091-2113, doi:10.5194/acp-13-2091-2013, 2013.

Shrivastava, M., Fast, J., Easter, R., Gustafson Jr., W. I., Zaveri, R. A., Jimenez, J. L., Saide, P., and Hodzic, A.: Modeling organic aerosols in a megacity: comparison of simple and complex representations of the volatility basis set approach, Atmos. Chem. Phys., 11, 6639-6662, doi:10.5194/acp-11-66392011, 2011.

Stavrakou, T., Müller, J.-F., De Smedt, I., Van Roozendael, M., Kanakidou, M., Vrekoussis, M., Wittrock, F., Richter, A., and
Burrows, J. P.: The continental source of glyoxal estimated by the synergistic use of spaceborne measurements and inverse modelling, Atmos. Chem. Phys., 9, 8431-8446, doi:10.5194/acp-98431-2009, 2009.

Thalman, R. and Volkamer, R.: Inherent calibration of a blue LED-CE-DOAS instrument to measure iodine oxide, glyoxal, methyl glyoxal, nitrogen dioxide, water vapour and aerosol extinction in open cavity mode, Atmos. Meas. Tech., 3, 1797-1814, doi:10.5194/amt-3-1797-2010, 2010.

Thalman, R. M., Waxman, E. M., Tyndall, G., Orlando, J., Karl, T., Kim, S., Seco, R., Su, L., Mak, J., and Volkamer, R.: Temperature dependent first generation product yields of minor mono and di-substituted carbonyls from the $\mathrm{OH}$-radical initiated oxidation of isoprene under high and near-zero $\mathrm{NO}_{\mathrm{x}}$ conditions, in preparation, 2013.

Tie, X., Madronich, S., Walters, S., Zhang, R., Rasch, P., and Collins, W.: Effect of clouds on photolysis and oxidants in the troposphere, J. Geophys. Res.-Atmos., 108, 4642, doi:10.1029/2003JD003659, 2003.

Trainic, M., Abo Riziq, A., Lavi, A., Flores, J. M., and Rudich, Y.: The optical, physical and chemical properties of the products of glyoxal uptake on ammonium sulfate seed aerosols, Atmospheric Chemistry and Physics, 11, 9697-707, doi:10.5194/acp11-9697-2011, 2011.

Volkamer, R., Platt, U., and Wirtz, K.: Primary and secondary glyoxal formation from aromatics: experimental evidence for the bicycloalkyl-radical pathway from benzene, toluene, and pxylene, J. Phys. Chem. A, 105, 7865-7874, 2001.

Volkamer, R., Molina, L., Molina, M., Shirley, T., and Brune, W.: DOAS measurement of glyoxal as an indicator for fast VOC chemistry in urban air, Geophys. Res. Lett., 32, L08806, doi:10.1029/2005GL022616, 2005.

Volkamer, R., Martini, F. S., Molina, L. T., Salcedo, D., Jimenez, J. L., and Molina, M. J.: A missing sink for gas-phase glyoxal in Mexico City: formation of secondary organic aerosol, Geophys. Res. Lett., 34, L19807, doi:10.1029/2007GL030752, 2007.

Volkamer, R., Ziemann, P. J., and Molina, M. J.: Secondary organic aerosol formation from acetylene $\left(\mathrm{C}_{2} \mathrm{H}_{2}\right)$ : Seed effect on SOA yields due to organic photochemistry in the aerosol aqueous phase, Atmos. Chem. Phys., 9, 1907-1928, doi:10.5194/acp9-1907-2009, 2009.

Warneke, C., Gouw, J. A., Edwards, P. M., Holloway, J. S., Gilman, J. B., Kuster, W. C., Graus, M., Atlas, E., Blake, D., Gentner, D. R., Goldstein, A. H., Harley, R. A., Alvarez, S., Rappenglück, B., Trainer, M., and Parrish, D.: Photochemical aging of volatile organic compounds in the Los Angeles basin: weekday-weekend effect, J. Geophys. Res.-Atmos., 118, 50185028, doi:10.1002/jgrd.50423, 2013.

Washenfelder, R. A., Langford, A. O., Fuchs, H., and Brown, S. S.: Measurement of glyoxal using an incoherent broadband cavity enhanced absorption spectrometer, Atmos. Chem. Phys., 8, 7779-7793, doi:10.5194/acp-8-7779-2008, 2008.

Washenfelder, R., Young, C., Brown, S., Angevine, W., Atlas, E., Blake, D., Bon, D., Cubison, M., De Gouw, J., Dusanter, S., Flynn, J., Gilman, J. B., Graus, M., Griffith, S., Grossberg, N., Hayes, P. L., Jimenez, J., Kuster, W., Lefer, B. L., Pollack, I., Ryerson, T., Stark, H., Stevens, P. S., and Trainer, M.: The glyoxal budget and its contribution to organic aerosol for Los Angeles, 
California, during CalNex 2010, J. Geophys. Res., 116, D00V02, doi:10.1029/2011JD016314, 2011.

Waxman, E. M., Dzepina, K., Ervens, B., Lee-Taylor, J., Aumont, B., Jimenez, J. L., Madronich, S., and Volkamer, R.: Secondary organic aerosol formation from semi-and intermediatevolatility organic compounds and glyoxal: relevance of $\mathrm{O} / \mathrm{C}$ as a tracer for aqueous multiphase chemistry, Geophys. Res. Lett., 40, 978-982, doi:10.1002/grl.50203, 2013.

Zaveri, R. A., Easter, R. C., and Peters, L. K.: A computationally efficient multicomponent equilibrium solver for aerosols (MESA), J. Geophys. Res.-Atmos., 110, D24203, doi:10.1029/2004JD005618, 2005.

Zaveri, R., Easter, R., Fast, J., and Peters, L.: Model for simulating aerosol interactions and chemistry (MOSAIC), J. Geophys. Res., 113, D13204, doi:10.1029/2007JD008782, 2008.

Zaveri, R. A., Shaw, W. J., Cziczo, D. J., Schmid, B., Ferrare, R. A., Alexander, M. L., Alexandrov, M., Alvarez, R. J., Arnott, W. P., Atkinson, D. B., Baidar, S., Banta, R. M., Barnard, J. C., Beranek, J., Berg, L. K., Brechtel, F., Brewer, W. A., Cahill, J. F., Cairns, B., Cappa, C. D., Chand, D., China, S., Comstock, J. M., Dubey, M. K., Easter, R. C., Erickson, M. H., Fast, J. D., Floerchinger, C., Flowers, B. A., Fortner, E., Gaffney, J. S., Gilles, M. K., Gorkowski, K., Gustafson, W. I., Gyawali, M., Hair, J., Hardesty, R. M., Harworth, J. W., Herndon, S., Hiranuma, N., Hostetler, C., Hubbe, J. M., Jayne, J. T., Jeong, H., Jobson, B. T., Kassianov, E. I., Kleinman, L. I., Kluzek, C., Knighton, B., Kolesar, K. R., Kuang, C., Kubátová, A., Langford, A. O., Laskin, A., Laulainen, N., Marchbanks, R. D., Mazzoleni, C., Mei, F., Moffet, R. C., Nelson, D., Obland, M. D., Oetjen, H., Onasch, T. B., Ortega, I., Ottaviani, M., Pekour, M., Prather, K. A., Radney, J. G., Rogers, R. R., Sandberg, S. P., Sedlacek, A., Senff, C. J., Senum, G., Setyan, A., Shilling, J. E., Shrivastava, M., Song, C., Springston, S. R., Subramanian, R., Suski, K., Tomlinson, J., Volkamer, R., Wallace, H. W., Wang, J., Weickmann, A. M., Worsnop, D. R., Yu, X.-Y., Zelenyuk, A., and Zhang, Q.: Overview of the 2010 Carbonaceous Aerosols and Radiative Effects Study (CARES), Atmos. Chem. Phys., 12, 7647-7687, doi:10.5194/acp-12-7647-2012, 2012.
Zhang, X., Liu, J., Parker, E. T., Hayes, P. L., Jimenez, J. L., Gouw, J. A., Flynn, J. H., Grossberg, N., Lefer, B. L., and Weber, R. J.: On the gas-particle partitioning of soluble organic aerosol in two urban atmospheres with contrasting emissions: 1. Bulk water-soluble organic carbon, J. Geophys. Res.-Atmos., 117, D00V16, doi:10.1029/2012JD017908, 2012.

Zhao, Y., Kreisberg, N. M., Worton, D. R., Isaacman, G., Weber, R. J., Liu, S., Day, D. A., Russell, L. M., Markovic, M. Z., VandenBoer, T. C., Murphy, J. G., Hering, S. V., and Goldstein, A. H.: Insights into secondary organic aerosol formation mechanisms from measured gas/particle partitioning of specific organic tracer compounds, Environ. Sci. Technol., 47, 3781-3787, 2013. 